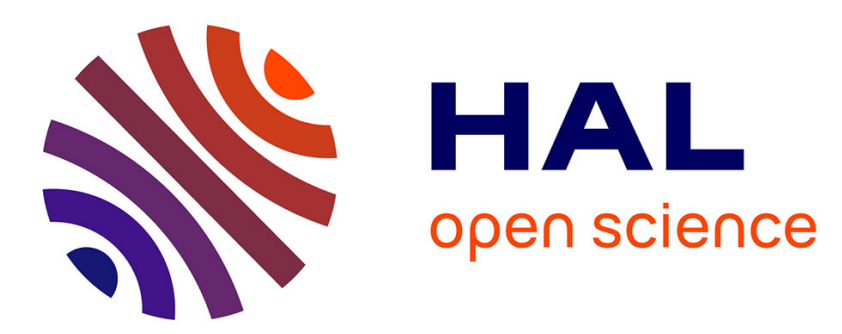

\title{
Étude de la lumière de fluorescence d'atomes excités en interaction résonnante avec un laser I. Analyse théorique
}

M. Dumont, B. Decomps

\section{To cite this version:}

M. Dumont, B. Decomps. Étude de la lumière de fluorescence d'atomes excités en interaction résonnante avec un laser I. Analyse théorique. Journal de Physique, 1968, 29 (2-3), pp.181-195. 10.1051/jphys:01968002902-3018100 . jpa-00206635

\section{HAL Id: jpa-00206635 https://hal.science/jpa-00206635}

Submitted on 1 Jan 1968

HAL is a multi-disciplinary open access archive for the deposit and dissemination of scientific research documents, whether they are published or not. The documents may come from teaching and research institutions in France or abroad, or from public or private research centers.
L'archive ouverte pluridisciplinaire HAL, est destinée au dépôt et à la diffusion de documents scientifiques de niveau recherche, publiés ou non, émanant des établissements d'enseignement et de recherche français ou étrangers, des laboratoires publics ou privés. 


\title{
ÉTUDE DE LA LUMIÈRE DE FLUORESGENGE D'ATOMES EXGITÉS EN INTERACTION RÉSONNANTE AVEG UN LASER ( $\left.{ }^{1}\right)$ I. ANALYSE THÉORIQUE
}

\author{
Par M. DUMONT et B. DECOMPS, \\ Faculté des Sciences de Paris, Laboratoire de Spectroscopie Hertzienne de l'E.N.S., associé au G.N.R.S.
}

(Reçu le 22 septembre 196\%.)

\begin{abstract}
Résumé. - On étudie l'effet de l'irradiation par un laser à gaz sur la lumière de fluorescence d'atomes excités par une décharge dans les niveaux supérieur et inférieur de la transition laser. Ces atomes sont soumis de plus à un champ magnétique statique. Le calcul, développé dans le formalisme des opérateurs tensoriels irréductibles, tient compte de l'existence de relaxations isotropes et du transfert d'excitation par émission spontanée entre le niveau supérieur et le niveau inférieur (effet de cascade). Suivant la méthode de Lamb [1], l'irradiation laser est décrite classiquement par son champ électrique et ses effets sont prévus par un développement en perturbation. On se limite ici à l'ordre 2. L'application des formules générales permet de définir un ensemble de grandeurs accessibles à l'expérience, rigoureusement décrites par l'ordre 2 des perturbations et simplement reliées aux valeurs des paramètres caractéristiques des diverses relaxations.

La vérification expérimentale sera décrite dans un second article. L'accord avec les résultats de l'analyse théorique présentée ici montre que cette méthode permet d'étudier effectivement plusieurs types de relaxations. L'application au niveau $2 p_{4}$ du néon y sera présentée.

Abstract. - The influence of a gas laser beam on the fluorescence light emitted by atoms excited by a discharge in the upper and lower levels of the laser transition is theoretically studied in the presence of a static magnetic field. Calculations are developed in the formalism of irreducible tensor operators. One takes into account the existence of isotropic relaxations and cascade effects due to spontaneous emission between the upper and the lower levels. Following Lamb [1], the laser beam is described classically by its electric field and its effects are calculated by perturbation theory. Second order formulae are applied to define a set of observables rigorously described by this second order theory and simply related to relaxation parameters.

In a second paper, experimental agreement with the present theoretical analysis will show the usefullness of this method to study several relaxation processes. Application to the $2 p_{4}$ level of neon will be described.
\end{abstract}

A. Introduction. - De nombreuses publications théoriques ou expérimentales ont traité le problème d'un laser à gaz soumis à un champ magnétique.

Si l'on s'intéresse au comportement des atomes à l'intérieur d'un tel laser, l'étude des caractéristiques du faisceau émis conduit à une interprétation délicate : en effet, il est sensible également aux propriétés de la cavité. De plus, du fait des saturations essentielles au fonctionnement d'un oscillateur, les caractéristiques du faisceau laser ne dépendent pas linéairement des paramètres atomiques.

L'utilisation de la lumière de fluorescence, proposée par Javan [2], pour l'étude des atomes en interaction avec l'onde laser évite ces complications. Depuis, de

(1) Cette étude a été réalisée avec l'aide de la D.R.M.E. nombreux expérimentateurs [3], [4] ont utilisé cette méthode. Il nous est apparu qu'une étude plus complète, théorique et expérimentale, était nécessaire à cause de l'existence de plusieurs types de relaxation et de l'influence de l'émission spontanée se produisant sur la même raie que l'oscillation laser.

Nous nous limitons pour l'instant à la réponse linéaire des atomes. Cela est suffisant pour atteindre les caractéristiques propres de chacun des deux niveaux atomiques pris séparément.

Plusieurs effets de saturation sont observables par cette méthode [5], [6]. L'étude détaillée de ces phénomènes fera l'objet d'une publication ultérieure.

1. DÉfinition du système ÉtudiÉ. - Nous considérons un gaz d'atomes possédant quatre niveaux d'énergie $a, b, f$ et $g$ (fig. 1). Nous cherchons à calculer 


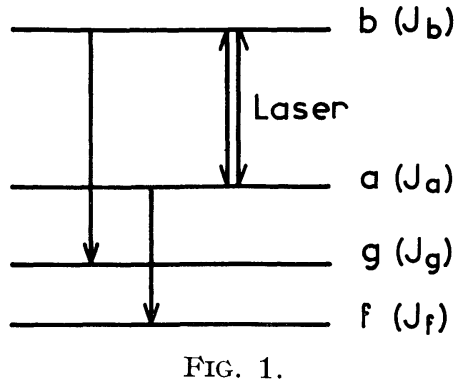

l'intensité et le diagramme de polarisation de la lumière de fluorescence émise par les atomes tombant du niveau $a$ sur le niveau $f$ ou par ceux tombant de $b$ sur $g$ lorsque le gaz est soumis :

a) à une décharge qui peuple tous les niveaux excités;

b) à une irradiation laser résonnante pour la transition $b \leftrightarrow a$;

c) à un champ magnétique statique.

2. Нy pothèses. - a) L'onde laser est indépendante du milieu atomique étudié : elle est imposée de l'extérieur. Le rayonnement laser, supposé multimode, sera traité classiquement comme un champ électrique oscillant [1].

b) Le milieu atomique. - 1) Les quatre niveaux considérés ont une structure Zeeman déterminée par leur moment cinétique total $J_{a}, J_{b}, J_{f}$ et $J_{g}$.

2) Les atomes du gaz sont soumis à l'agitation thermique et nous supposons que la répartition des vitesses est une répartition de Maxwell de température $T$ indépendante de l'état d'excitation interne des atomes.

3) La décharge est supposée homogène sur tout le volume étudié et constante dans le temps.

4) Elle n'introduit aucune orientation ni aucun alignement dans les niveaux excités. Ceci revient à admettre qu'aux pressions utilisées le bombardement des atomes par les électrons est isotrope.

5) Nous supposerons que les mécanismes de relaxation (collisions, diffusion multiple) ont la symétrie sphérique, c'est-à-dire qu'il n'y a aucune direction privilégiée. Cela signifie que, lors d'une collision, la vitesse relative des deux atomes a la même probabilité d'avoir n'importe quelle direction. Cela signifie également, pour la diffusion multiple, qu'un photon émis par un atome a la même probabilité d'être réabsorbé quelle que soit la direction dans laquelle il est émis. En fait, aucune de ces deux conditions n'est réalisée rigoureusement : d'une part, le laser n'interagit qu'avec des atomes de vitesse déterminée pour lesquels les collisions ne sont sûrement pas isotropes, d'autre part, la cellule a la forme d'un tube mince et long, et la diffusion multiple n'est plus isotrope dès que le taux de réabsorption de la lumière devient faible.

Nous avons étudié cette symétrie axiale par une théorie phénoménologique, mais les expériences n'ont pu mettre en évidence les effets prévus. Donc, à la précision de nos mesures, la théorie isotrope suffit, ce qui nous permet de décomposer la matrice densité en opérateurs tensoriels irréductibles et d'attribuer un seul temps de relaxation par ordre d'opérateur [8].

6) Nous ne tiendrons pas compte d'éventuels changements de vitesse des atomes lors des collisions. Cette hypothèse n'apporte d'ailleurs aucune modification importante pour le calcul au second ordre auquel nous nous limiterons ici.

7) Enfin, nous tiendrons compte des atomes tombant de $b$ en $a$ par émission spontanée.

3. Plan du calgul. - Nous commencerons par poser l'équation de Schrödinger à laquelle obéit la matrice densité d'un paquet d'atomes de vitesse $v$ $(\S \mathrm{B})$.

Cette équation sera résolue par un traitement de perturbation [1], [7] $(\S \mathrm{C})$. Au second ordre de ce développement, nous trouverons la partie linéaire de la modification du système atomique sous l'effet de l'irradiation laser.

$\mathrm{Au}$ quatrième ordre (non traité ici), nous pourrions trouver les premiers effets non linéaires (saturation).

Après intégration sur les vitesses des atomes, nous obtenons la matrice densité globale de la vapeur et nous en déduisons $(\S \mathrm{D})$ le diagramme de rayonnement de la lumière de fluorescence.

Enfin, nous montrons $(\S \mathrm{E})$ sur un cas particulier comment cette étude permet de définir un ensemble de grandeurs accessibles à l'expérience qui permettent de mesurer les valeurs des temps de relaxation.

B. Equation du problème. - En raison de l'effet Doppler, des atomes de vitesse différente ne voient pas la même fréquence optique; ils ne sont donc pas soumis au même hamiltonien. Pour cette raison, nous sommes obligés de résoudre le problème séparément pour chaque classe de vitesse.

En fait, seule compte la projection $v$ de la vitesse des atomes sur la direction de propagation de l'onde laser.

De même, il suffit de repérer la position d'un atome par son abscisse $r$ le long de l'axe du laser :

$$
v=\frac{\mathbf{v k}}{|k|}, \quad r=\frac{\mathbf{r k}}{|k|}
$$

si $\mathbf{k}$ est le vecteur d'onde du laser $(r$ est la position par rapport aux plans équiphases).

Considérons donc le paquet d'atomes de vitesse $v$ passant en $r$ à l'instant $t$. Il est représenté par la matrice densité $\rho(v, r, t)$. $r$ et $t$ sont repérés par rapport aux axes du laboratoire. Pour connaître cette matrice densité, nous devons résoudre l'équation de Schrödinger dans un système d'axes en mouvement avec le paquet d'atomes considéré : à l'instant $t^{\prime}=t+\tau$, les atomes se trouvent en $r^{\prime}=r+v \tau$. Dans le sys- 
tème d'axes en mouvement, l'équation de Schrödinger s'écrit (avec $\hbar=1)$ :

$$
\begin{aligned}
\frac{\mathrm{d}}{\mathrm{d} \tau} \rho(v, r+v \tau, t & +\tau) \\
=-i[ & \mathscr{H}(r+v \tau, t+\tau), \rho(v, r+v \tau, t+\tau)] \\
& +\Lambda(v)+\left(\frac{\mathrm{d} \rho}{\mathrm{d} \tau}\right)_{\text {relax. }}+\left(\frac{\mathrm{d} \rho}{\mathrm{d} \tau}\right)_{\text {tr. }}
\end{aligned}
$$

que nous écrirons de façon plus simple :

$$
\begin{aligned}
& \dot{\rho}\left(v, r^{\prime}, t^{\prime}\right)=-i\left[\mathscr{H}\left(r^{\prime}, t^{\prime}\right), \rho\left(v, r^{\prime}, t^{\prime}\right)\right] \\
& +\Lambda(v)+\left(\frac{\mathrm{d} \rho}{\mathrm{d} t^{\prime}}\right)_{\text {relax. }}+\left(\frac{\mathrm{d} \rho}{\mathrm{d} t^{\prime}}\right)_{\text {tr. }} .
\end{aligned}
$$

L'hamiltonien $\mathscr{H}$ décrit l'évolution propre de $\rho$ ainsi que l'action du champ magnétique et de l'onde laser. $\Lambda$ représente l'excitation par la décharge supposée homogène (hypothèse 3$))$. $(\mathrm{d} \rho / \mathrm{d} t)_{\text {relax. }}$ représente toutes les relaxations et $(\mathrm{d} \rho / \mathrm{d} t)_{\text {tr. }}$ le transfert d'atomes de $b$ en $a$ par émission spontanée. Lorsque cette équation sera résolue, il suffira de poser $t=t^{\prime} \quad(\tau=0)$ pour obtenir $\rho(v, r, t)$ dans le système du laboratoire.

Explicitons les éléments de cette équation :

1. La matrice densité p. - Nous ne représentons par cette matrice que les atomes se trouvant dans les niveaux $a$ et $b$. Il en résulte que la trace de $\rho$ (nombre d'atomes en $a$ ou $b$ ) n'est pas constante.

Dans la base standard $|J M\rangle$, les éléments de matrice seront notés :

$$
\rho_{M_{a} M_{b}}=\left\langle J_{a} M_{a}|\rho| J_{b} M_{b}\right\rangle
$$

$\rho$ est formée de quatre sous-matrices :

$$
\rho=\left(\begin{array}{cc}
a a \rho & a b \rho \\
b a \rho & b b \rho
\end{array}\right) \text {. }
$$

Les sous-matrices ${ }_{a b} \rho$ et ${ }_{b a} \rho$ sont formées de ce que nous appellerons les éléments de «cohérence optique ».

${ }_{a a} \rho$ et ${ }_{b b} \rho$ contiennent des termes diagonaux ou « populations » et des termes non diagonaux ou « cohérences Zeeman ».

Pour effectuer le calcul, il est commode de décomposer $\rho$ sur une base d'opérateurs tensoriels irréductibles normalisés :

$(\alpha, \beta=a$ ou $b)$

$$
{ }_{\alpha \beta} \rho(v, r, t)=\sum_{k, q}{ }_{\alpha \beta} \rho_{q}^{k}(v, r, t) \cdot_{\alpha \beta} T_{q}^{(k)}
$$

avec : $\quad \quad \alpha \beta \rho_{q}^{k}=\operatorname{Tr}\left(\rho \cdot{ }_{\alpha \beta} T_{q}^{(k)+}\right)$.

Rappelons quelques relations utiles :

$$
\begin{gathered}
{ }_{\alpha \beta} T_{q}^{(k)+}=(-)^{J_{\alpha}-J_{\beta}+q}{ }_{\beta \alpha} T_{-q}^{(k)} \\
\operatorname{Tr}\left({ }_{\alpha \beta} T_{q}^{(k)} \cdot \alpha^{\prime} \beta^{\prime} T_{q^{\prime}}^{\left(k^{\prime}\right)+}\right)=\delta_{\alpha \alpha^{\prime}} \cdot \delta_{\beta \beta^{\prime}} \cdot \delta_{k k^{\prime}} \cdot \delta_{q q^{\prime}}
\end{gathered}
$$

enfin l'élément de matrice réduit est :

$$
\left\langle J_{\alpha}\left\|_{\alpha \beta} T^{(k)}\right\| J_{\beta}\right\rangle=\sqrt{2 k+1} .
$$

Pour simplifier, nous écrirons $\alpha_{\alpha} \rho,{ }_{\alpha} T$ à la place de ${ }_{\alpha \alpha} \rho,{ }_{\alpha \alpha} T$.

2. La matrice D'EXaitation $\Lambda(v)$. - Elle représente les atomes portés par unité de temps et de volume dans les états $a$ ou $b$ par la décharge. Suivant l'hypothèse 3 ), $\Lambda$ est diagonale dans la base standard $|J M\rangle$. En notation d'opérateurs, $\Lambda$ est multiple de l'opérateur scalaire :

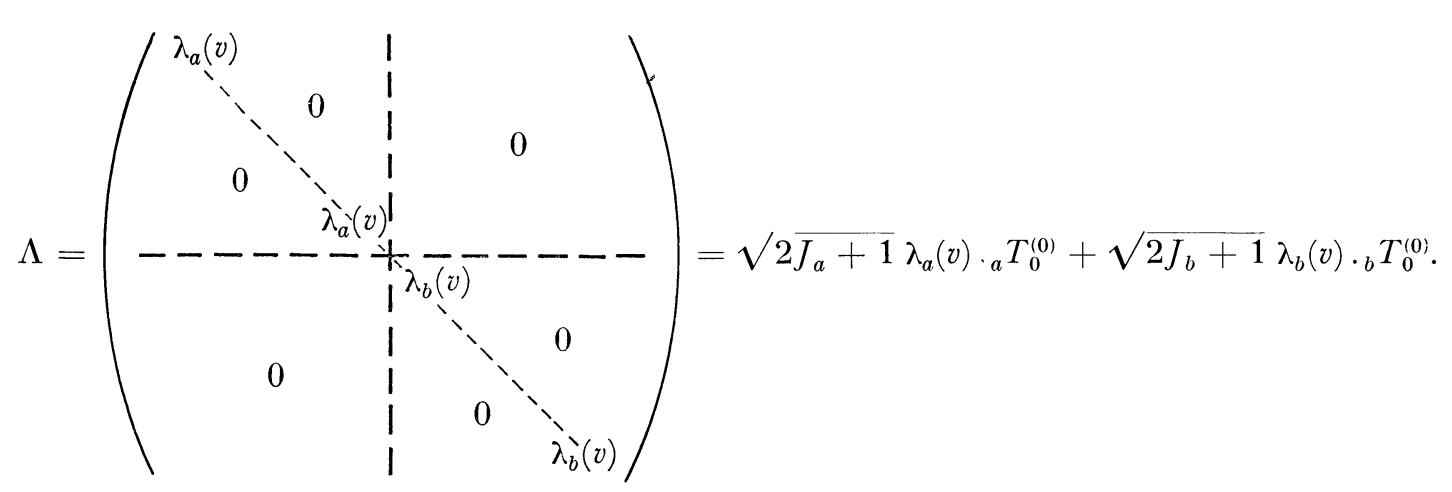

D'après l'hypothèse 2) :

$$
\lambda_{\alpha}(v)=\lambda_{\alpha} \frac{1}{u \sqrt{ } \pi} \mathrm{e}^{-(v / u)^{2}}
$$

avec :

$$
u=\sqrt{2 R T / M}
$$

suivant la loi de Maxwell de la théorie cinétique des gaz.

3. Le terme de Relaxation $\left(\mathrm{d} \rho / \mathrm{d} t^{\prime}\right)_{\text {relax, }}$ - Ce terme contient la durée de vie radiative de chacun des niveaux ainsi que l'effet des collisions et éventuel- lement de la diffusion multiple des raies de fluorescence.

Suivant l'hypothèse d'isotropie 5), on a, en absence de diffusion multiple :

$$
\left(\frac{\mathrm{d}_{\alpha \beta} \rho_{q}^{k}}{\mathrm{~d} t}\right)_{\text {relax. }}=-\Gamma_{\alpha \beta}(k)_{\alpha \beta} \rho_{q}^{k}
$$

$$
\Gamma_{a b}(k)=\Gamma_{b a}(k)=\gamma_{a}+\gamma_{b}+\left(\Gamma_{a b}(k)\right)_{\text {coll. }}
$$

$\Gamma_{a}(k)=\gamma_{a}+\left(\Gamma_{a}(k)\right)_{\text {coll. }} ; \quad \Gamma_{b}(k)=\gamma_{b}+\left(\Gamma_{b}(k)\right)_{\text {coll. }}$ 
$\tau_{a}=1 / \gamma_{a}$ et $\tau_{b}=1 / \gamma_{b}$ sont les durées de vie radiatives des niveaux $a$ et $b . \quad\left(\Gamma_{a b}(k)\right)_{\text {coll., }}\left(\Gamma_{a}(k)\right)_{\text {coll. }}$. et $\left(\Gamma_{b}(k)\right)_{\text {coll. }}$ représentent l'effet des collisions; ces termes sont proportionnels à la pression.

S'il y a de la diffusion multiple sur les raies de fluorescence émises par $a$ (ou $b$ ), il faut remplacer (I .11) par :

$\Gamma_{a}(k)=\left(\Gamma_{a}(k)\right)_{\text {coll, }}+\sum_{i} \gamma_{a i}\left(1-\alpha_{a}(k, i) x_{i}\right)$

L'indice $i$ représente tous les niveaux vers lesquels l'atome peut effectuer une transition radiative à partir du niveau $a . \gamma_{a i}$ est la probabilité de chaque transition et $x_{i}$ le taux de réabsorption de la raie (probabilité, pour chaque photon, d'être réabsorbé); $\alpha_{a}(k, i)$ est un coefficient dont la valeur est tabulée [9], [10] en fonction de $k, J_{a}$ et $J_{i}$.

Cette façon d'écrire la diffusion multiple n'est valable que pour le calcul au deuxième ordre que nous faisons ici. En effet, l'atome 2 qui absorbe le photon émis par l'atome 1 n'a pas en général la même vitesse que l'atome 1 (seules les projections de $\mathbf{v}_{1}$ et $\mathbf{v}_{2}$ sur la droite joignant les deux atomes sont égales). Il en résulte que l'atome 2 n'interagit plus, en général, avec le laser et ne participe pas aux effets d'ordre supérieur.
4. Le TERME DE TRANSFERT $(\mathrm{d} \rho / \mathrm{d} t)_{\mathrm{tr}}$. - Ce terme représente l'effet sur $a$ de l'émission spontanée de $b$ vers $a$. Nous avons déjà tenu compte de la relaxation de $b$ par ce processus dans $(\mathrm{d} \rho / \mathrm{d} t)_{\text {relax. }} \cdot(\mathrm{d} \rho / \mathrm{d} t)_{\mathrm{tr}}$. n'agit donc que sur le niveau $a$. Il introduit dans les équations un terme source dépendant de ${ }_{b} \rho$.

En raison du caractère isotrope de l'émission spontanée, on peut montrer que le couplage entre $a$ et $b$ ne se fait qu'entre tenseurs de même nature (même $k$ et même q) [11], [12] :

$$
\begin{aligned}
\left(\frac{\mathrm{d}_{a} \rho_{q}^{k}}{\mathrm{~d} t}\right)_{\mathrm{tr} .} & =(-)^{J_{a}+J_{b}+k+1} \gamma_{b a}\left(2 J_{b}+1\right) \\
& \left.=\Theta(b, a, k)_{b \rho_{q}^{k} .} \begin{array}{ccc}
k & J_{b} & J_{b} \\
1 & J_{a} & J_{a}
\end{array}\right\}_{b} \rho_{q}^{k}
\end{aligned}
$$

En l'absence d'irradiation laser, ce processus ne peut se distinguer des autres processus de population du niveau $a$ et il est déjà inclus dans $\lambda_{a}(v)$. Autrement dit, le terme $\left(\mathrm{d}_{a} \rho_{q}^{k} / \mathrm{d} t\right)_{\text {tr. }}$ ne joue que sur la variation de $\rho$ due à l'irradiation laser.

5. L'hamiltonien :

$$
\mathscr{H}=\mathscr{H}_{0}-M_{Z} H+R(r, t)
$$

a) $\mathscr{H}_{0}$ est l'hamiltonien de l'atome isolé :



b) $\mathscr{H}_{Z}=-M_{Z} H$ est l'hamiltonien Zeeman.

L'axe $\mathrm{Oz}$ a été choisi par définition parallèle au champ magnétique. A l'intérieur de chacun des niveaux $a$ et $b$, le moment magnétique est proportionnel à $\mathbf{J}$ :

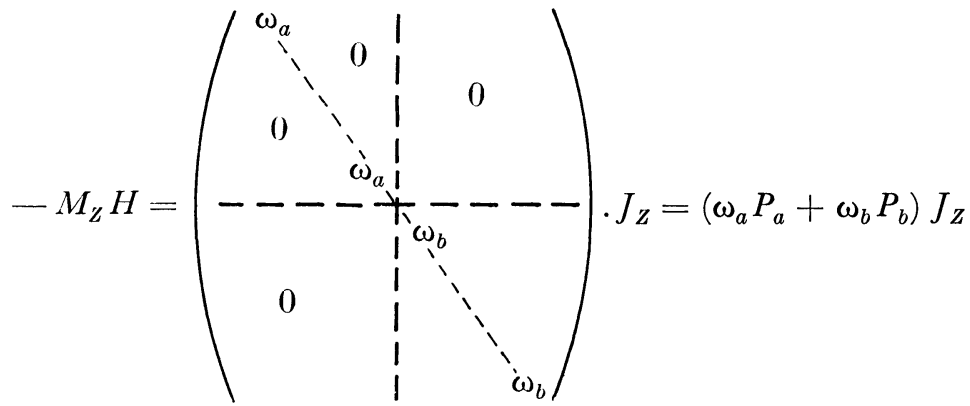

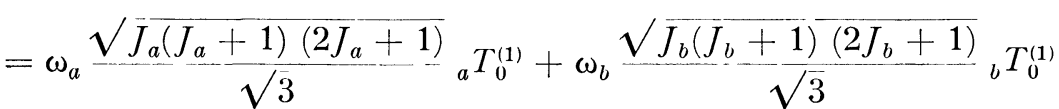


où $\omega_{\alpha}=g_{\alpha} \beta H$ est l'écart Zeeman $(\beta=$ magnéton de Bohr, $g_{\alpha}=$ facteur de Landé). $P_{\alpha}$ est le projecteur sur le niveau $\alpha$.

c) L'hamiltonien d'interaction avec le champ électrique $\mathbf{E}$ du faisceau laser s'écrit :

$$
R(r, t)=-\mathbf{P E}(r, t)
$$

(approximation dipolaire électrique), $\mathbf{P}$ est l'opérateur dipôle électrique.

$$
\begin{aligned}
\mathbf{E}(r, t) & =\sum_{\mu} \mathbf{E}^{\mu}(r, t) \\
= & \sum_{\mu}\left(\mathscr{E}^{\mu} \mathrm{e}^{-i\left(\omega_{\mu} t-k_{\mu} r\right)}+\mathscr{E}^{\mu^{*}} \mathrm{e}^{+i\left(\omega_{\mu} t-k_{\mu} r\right)}\right)
\end{aligned}
$$

l'indice $\mu$ sert à distinguer les différents modes de la raie laser. Avec cette notation, nous pouvons représenter une onde lumineuse de polarisation quelconque. $\mathscr{E}^{\mu}$ contient la phase relative des différents modes. En particulier, pour une polarisation plane, on a :

$$
\mathscr{E}^{\mu}=\mathbf{E}^{\mu} \mathrm{e}^{-i \varphi_{\mu}} \quad \text { avec } \quad \mathbf{E}^{\mu} \text { réel. }
$$

Pour représenter l'onde stationnaire à l'intérieur du laser, il suffit, dans la sommation sur $\mu$, d'associer deux ondes progressives à chaque mode :

$$
\omega_{\mu}=\omega_{\mu^{\prime}} ; \quad k_{\mu}=-k_{\mu^{\prime}} \text {. }
$$

Nous pouvons exprimer $R$ en fonction des composantes standard de l'opérateur $\mathbf{P}$ et du vecteur $\mathbf{E}$ :

$$
\begin{aligned}
& E_{0}=E_{z} ; \quad E_{ \pm 1}=\mp \frac{1}{\sqrt{2}}\left(E_{x} \pm i E_{y}\right) \quad \text { (I.19) } \\
& R(r, t)=-\sum_{q}(-)^{q} P_{q} E_{-q} \\
& =-\sum_{\mu q}(-)^{q} P_{q}\left[\mathscr{E}_{-q} \mathrm{e}^{-i\left(\omega_{\mu} t-k_{\mu} r\right)}+\left(\mathscr{E}^{\mu^{*}}\right)_{-q} \mathrm{e}^{i\left(\omega_{\mu} t-k_{\mu} r\right)}\right] .
\end{aligned}
$$

Comme l'opérateur dipôle électrique, $R$ n'a pas d'éléments de matrice à l'intérieur de chacun des niveaux $a$ ou $b$ (opérateur impair) :

$$
R=\left(\begin{array}{c:c}
0 & a b \\
\hdashline & 0
\end{array}\right) ; \quad{ }_{a b} R={ }_{b a} R^{+} .
$$

Remarque : On introduit couramment [13] comme vecteurs de base les polarisations $\sigma^{+}, \sigma^{-}$et $\pi$ qui s'écrivent respectivement :

$$
\begin{gathered}
\mathbf{e}_{+1}=-\frac{1}{\sqrt{2}}\left(\mathbf{e}_{x}+i \mathbf{e}_{y}\right) \\
\mathbf{e}_{-1}=\frac{1}{\sqrt{2}}\left(\mathbf{e}_{x}-i \mathbf{e}_{y}\right) ; \quad \mathbf{e}_{0}=\mathbf{e}_{z} .
\end{gathered}
$$

Avec cette base, on écrit :

$$
\mathbf{E}=\sum_{q}(-)^{q} E_{-q} \mathbf{e}_{q} \text {. }
$$

Par définition :

$$
\mathscr{E}^{*}=\sum_{i} \mathscr{E}_{, i}^{*} \mathbf{e}_{\imath} \quad(i=x, y, z)
$$

On trouve alors :

$$
\left(\mathscr{E}^{*}\right)_{q}=(-)^{q}\left(\mathscr{E}_{-q}\right)^{*} \quad(q=0, \pm 1) .
$$

G. Résolution des équations. - 1. MÉthode D'APPRoximation. - En général, nous ne pouvons pas résoudre rigoureusement l'équation (I.1). Nous devons utiliser un calcul de perturbation (Lamb [1], Bloembergen [7]).

Nous cherchons un développement de la matrice $\rho$ en fonction des puissances successives du champ électrique de l'onde laser.

Les ordres successifs peuvent être calculés par itération :

$$
\begin{aligned}
{ }^{(0)} \dot{\rho} & =-i\left[\mathscr{H}_{0}+\mathscr{H}_{Z},{ }^{(0)} \rho\right]+\left(\frac{\mathrm{d}^{(0)} \rho}{\mathrm{d} t}\right)_{\text {relax. }}+\Lambda \\
{ }^{(1)} \dot{\rho} & =-i\left[\mathscr{H}_{0}+\mathscr{H}_{Z},{ }^{(1)} \rho\right]+\left(\frac{\mathrm{d}^{(1)} \rho}{\mathrm{d} t}\right)_{\text {relax. }+\mathrm{tr.}}-i\left[R,{ }^{(0)} \rho\right] \\
& \vdots \\
{ }^{(n)} \dot{\rho} & =-i\left[\mathscr{H}_{0}+\mathscr{H}_{Z},{ }^{(\mathrm{n})} \mathrm{\rho}\right]+\left(\frac{\mathrm{d}^{(\mathrm{n})} \mathrm{\rho}}{\mathrm{d} t}\right)_{\text {relax. }+\mathrm{tr} .}-i\left[R,{ }^{(n-1)} \rho\right] .
\end{aligned}
$$

En raison de ce qui a été dit au $\$$ B.4, le terme de transfert n'existe pas à l'ordre $(0)$, car il est inclus dans $\Lambda$.

Nous ne cherchons que la solution stationnaire de ces équations.

A l'ordre $(0)$, le terme source $\Lambda$ est scalaire, la solution stationnaire de (0) $\rho$ l'est donc aussi. (0) $\rho$ nc contient pas de cohérences optiques (ni Zeeman).

A l'ordre (1), le terme source est de la forme ${ }_{a b} R_{b}^{(0)} \rho$, dont ${ }^{(1)} \rho$ n'aura que des termes de cohérence optique. Seuls $\stackrel{(1)}{a b} \rho={ }_{b a}^{(1)} \rho^{+}$seront différents de zéro. 
Ainsi de suite, on montre que :

$$
{ }_{a}^{(2 n+1)} \rho={ }_{b}^{(2 n+1)} \rho=\underset{a b \rho}{(2 n)}=\underset{b a \rho}{(2 n)}=0 .
$$

Aux ordres pairs, nous n'avons que des « cohérences Zeeman » et des populations. Aux impairs, nous n'avons que des « cohérences optiques ».
Pour résoudre les équations (I.23), nous allons utiliser le développement (I.2) de la matrice densité sur la base des opérateurs tensoriels irréductibles :

$$
{ }_{\alpha \beta}^{(\mathrm{n})} \dot{\rho}_{q}^{k}=\operatorname{Tr}\left({ }^{(\mathrm{n})} \dot{\rho}_{\alpha \beta} T_{q}^{(k)+}\right) .
$$

Le calcul de $\operatorname{Tr}\left(\left[\mathscr{H}_{0}+\mathscr{H}_{Z},{ }^{(n)} \rho\right]_{\alpha \beta} T_{q}^{(k)+}\right)$ nous conduit aux équations :

(a) $\quad{ }_{a}^{(2 n)} \dot{\rho}_{q}^{k}=-\left(i q \omega_{a}+\Gamma_{a}(k)\right){ }_{a}^{(2 n)} \rho_{q}^{k}+\Theta(b, a, k){ }_{b}^{(2 n)} \rho_{q}^{k}-i \operatorname{Tr}\left[\left({ }_{a b} R R_{b a}^{(2 n-1)}-{ }_{a b}^{(2 n-1)}{ }_{b a} R\right){ }_{a} T_{q}^{(k)+}\right]$

$$
{ }_{b}^{(2 \mathrm{n})} \dot{\rho}_{q}^{k}=-\left(i q \omega_{b}+\Gamma_{b}(k)\right){ }_{b}^{(2 n)} \rho_{q}^{k}-i \operatorname{Tr}\left[\left({ }_{b a} R \underset{a b \rho}{(2 n-1)}-{ }_{b a}^{(2 n-1)} \rho{ }_{a b} R\right){ }_{b} T_{q}^{(k)+}\right]
$$

$$
\begin{aligned}
& { }_{a b}^{(2 \mathbf{n}+1)} \dot{\rho}_{q}^{k}=\left[i\left(\omega-q \frac{\omega_{a}+\omega_{b}}{2}\right)-\Gamma_{a b}(k)\right]{ }_{a b}^{(2 n+1)} \rho_{q}^{k}-i \operatorname{Tr}\left[\left({ }_{a b} R^{(2 n)} \rho-{ }_{a}^{(2 n)} \rho_{a b} R\right){ }_{a b} T_{q}^{(k)+}\right] \\
& -i \frac{\omega_{a}-\omega_{b}}{2}\left[q \frac{J_{a}\left(J_{a}+1\right)-J_{b}\left(J_{b}+1\right)}{k(k+1)} \underset{a b \rho_{q}^{k}}{(2 n+1)} \rho_{a b}^{k} \underset{a b}{(2 n+1)} \rho_{q}^{k-1}+a(k+1){ }_{a b \rho_{q}}^{(2 n+1)}\right]
\end{aligned}
$$

avec :

$$
\omega=W_{b}-W_{a}
$$

et : $\quad a(k)=\frac{1}{k} \sqrt{\frac{\left(J_{a}+J_{b}+k+1\right)\left(J_{a}-J_{b}+k\right)\left(-J_{a}+J_{b}+k\right)\left(J_{a}+J_{b}+1-k\right)\left(k^{2}-q^{2}\right)}{(2 k-1)(2 k+1)}}$.

Les équations $(\mathrm{I} .25, b)$ sont très faciles à résoudre puisque ce sont des équations différentielles indépendantes. Une fois ces équations résolues, il suffit d'en reporter les solutions dans les équations (I.25,a), également indépendantes les unes des autres.

Par contre, (I.25, c) est un système différentiel. Le terme de couplage entre les différents ordres d'opérateurs exprime le fait que, dans le cas général, les composantes $\sigma^{+}, \sigma^{-}$et $\pi$ de la raie optique ne sont pas simples (effet Zeeman anormal). Chaque élément de matrice $\rho_{M_{a} M_{b}}$ a une fréquence particulière de précession libre $\left(\omega+M_{b} \omega_{b}-M_{a} \omega_{a}\right)$. Les ${ }_{a b} \rho_{a}^{k}$ de même $q$, mais de $k$ différents sont des combinaisons linéaires de tous les éléments $\rho_{M_{a} M_{b}}$ tels que :

$$
q=M_{a}-M_{b}
$$

ce qui explique qu'ils n'ont pas de fréquence de précession propre et que leurs équations sont couplées.

Il y a deux cas simples où ces couplages disparaissent :

- L'un des $J$ est nul (effet Zeeman normal);

- Les deux niveaux ont même facteur de Landé.

Bien que le système différentiel soit soluble dans chaque cas particulier, nous supposerons, pour simplifier le calcul, que nous sommes dans l'un des deux cas précédents. En effet, la raie laser $1,52 \mu$ du néon correspond au premier cas et la raie $6328 \AA$ corres- pond presque au second : $g_{a}-g_{b} \sim 5 \times 10^{-3} g_{a}$. Tant que le champ magnétique n'est pas trop fort $\left(<100\right.$ gauss), $\omega_{b}-\omega_{a}$ est négligeable devant $\Gamma_{a}$.

Enfin, pour les effets linéaires ( $2^{\mathrm{e}}$ ordre), nous montrerons a posteriori que l'approximation reste valable tant que $\omega_{b}-\omega_{a} \ll \Delta \nu$ (largeur Doppler).

2. Calgul a l'ordre 0. - Comme nous l'avons déjà dit, seule la composante scalaire de ${ }^{(0)} \rho$ est non nulle. La solution stationnaire est :

$$
{ }_{\alpha}^{(0)} \rho_{0}^{0}(v, r, t)=\sqrt{2 J_{\alpha}+1} \lambda_{\alpha}(v) / \Gamma_{\alpha}(0)
$$$$
\text { ou } \quad{ }^{(0)} \rho_{M_{\alpha} M_{\alpha}}=\lambda_{\alpha}(v) / \Gamma_{\alpha}(0) \text {. }
$$

3. ORdRe (1). - On a en posant :

$$
\omega_{Z}=\frac{\omega_{a}+\omega_{b}}{2}\left(\text { ou } \omega_{Z}=\omega_{a, b} \text { si } J_{b, a}=0\right)
$$

${ }_{a b}^{(1)} \dot{\rho}_{q}^{k}=\left[-\Gamma_{a b}(k)+i\left(\omega-q \omega_{Z}\right)\right]_{a b}^{(1)} \rho_{q}^{k}$

$$
+i\left[\frac{1}{\sqrt{2 J_{a}+1}}{ }_{a}^{(0)} \rho_{0}^{0}-\frac{1}{\sqrt{2} \bar{J}_{b}+1}{ }_{b}^{(0)} \rho_{0}^{0}\right] \operatorname{Tr}\left(R_{a b} T_{q}^{(k)+}\right)
$$

$R$ est formé de l'opérateur dipôle électrique qui est proportionnel à l'opérateur tensoriel d'ordre (1), $T^{(1)}$, Il résulte alors de la relation de normalisation (I.5) que ${ }_{a b}^{(1)}$ n'a que les trois composantes $k=1 ; q=0$. \pm 1 :

$$
\begin{aligned}
& \frac{\mathrm{d}}{\mathrm{d} \tau}{ }^{(1)}{ }^{(1)} \rho_{u}^{1}(v, r+v \tau, t+\tau)=\left[-\Gamma_{a b}(1)+i\left(\omega-q \omega_{Z}\right){ }_{a b}^{(1)} \rho_{q}^{k}(v, r+v \tau, t+\tau)\right. \\
& +i N(v) \frac{P_{a b}}{\sqrt{3}} \sum_{\mu}\left[(-)^{q} \mathscr{E}_{-q}^{\mu_{\alpha}} \mathrm{e}^{-i\left[\omega_{\mu}(t+\tau)-k_{\mu}(r+v \tau)\right]}+\mathscr{E}_{q}^{\mathscr{U}^{*}} \mathrm{e}^{+i[\ldots]}\right]
\end{aligned}
$$


où $P_{a b}=\left\langle J_{a}\|P\| J_{b}\right\rangle$ est l'élément de matrice réduit de l'opérateur $P$ et $N(v)=\frac{\lambda_{b}(v)}{\Gamma_{b}(0)}-\frac{\lambda_{a}(v)}{\Gamma_{a}(0)}$ est l'inversion de population (entre les sous-niveaux de $a$ et de $b$ ) pour les atomes de vitesse $v$.

La solution stationnaire de l'équation (I .28) est la somme des oscillations forcées correspondant à chacun des termes sources :

$$
\begin{aligned}
&{ }_{a b}^{(1)} P_{q}^{1}=i N(v) \frac{\left\langle J_{a}\|P\| J_{b}\right\rangle}{\sqrt{3}} \\
& \sum_{\mu} \frac{\mathscr{E}_{q} \mu^{*}}{\mathrm{e}^{i\left(\omega_{\mu} t-k_{\mu} r\right)}} \frac{}{\Gamma_{a b}(1)-i\left(\omega-q \omega_{Z}-\omega_{\mu}+k_{\mu} v\right)}
\end{aligned}
$$



Nous avons abandonné les termes de fréquence négative (approximation séculaire) qui auraient donné des termes en $\left[\Gamma_{a b}(1)-i\left(\omega-q \omega_{a}+\omega_{\mu}-k_{\mu} v\right)\right]^{-1}$. Ces termes sont parfaitement négligeables aux fréquences optiques $\left(\omega \sim 2 \pi \times 10^{15}\right)$.

A l'ordre (1), ces formules nous permettraient de calculer la polarisation moyenne du gaz, d'où l'indice et l'absorption (linéaire).

4. ORdRe (2). - Nous commencerons par calculer $\underset{b}{(2)} \rho$ :

$$
\begin{aligned}
{ }_{b}^{(2)} \dot{\rho}_{q}^{k}=-\left(i q \omega_{b}+\right. & \left.\Gamma_{b}(k)\right) \underset{b}{(2)} \rho_{q}^{k} \\
& -i \operatorname{Tr}\left(\left[R,{ }^{(1)} \rho\right]_{b} T_{q}^{(k)+}\right) .
\end{aligned}
$$

Calculons le terme source :

$\operatorname{Tr}\left(\left[R,{ }^{(1)} \rho\right]_{b} T_{q}^{(k)+}\right)=\operatorname{Tr}\left[\left({ }_{b a} R \underset{a b \rho}{(1)} \rho-{ }_{b a \rho}^{(1)} R_{a b}\right){ }_{b} T_{q}^{(k)+}\right]$

$$
\begin{aligned}
& =-\sum_{q_{1} q_{2}}\left[(-)^{q_{2}} \frac{P_{b a}}{\sqrt{3}} E_{-q_{2}} \stackrel{(1)}{a b} \rho_{q_{1}}^{1}-(-)^{q_{1}} \frac{P_{a b}}{\sqrt{3}} E_{-q_{1} b a q_{q_{2}}}^{(1)} \rho_{q^{1}}^{1} \operatorname{Tr}\left({ }_{b a} T_{q_{2} a b}^{(1)} T_{q_{1} b}^{(1)} T_{q}^{(k)+}\right)\right. \\
& \operatorname{Tr}\left({ }_{b a} T_{q_{2} a b}^{(1)} T_{q_{1} b}^{(1)} T_{q}^{(k)+}\right)=(-)^{2 J_{a}} 3\left\langle 11 q_{1} q_{2} \mid k q\right\rangle\left\{\begin{array}{ccc}
k & 1 & 1 \\
J_{a} & J_{b} & J_{b}
\end{array}\right\} .
\end{aligned}
$$

En explicitant $E_{q}$ et ${ }^{(1)} \rho$ et en ne gardant que les faibles fréquences du type $\omega_{\nu}-\omega_{\mu}$ (nouvelle approximation séculaire), on obtient :

$$
\begin{aligned}
& -i \operatorname{Tr}\left(\left[R,{ }^{(1)} \rho\right]_{b} T_{q}^{(k)+}\right)=\sum_{\nu \mu} B_{q}^{k}\left(v, \nu, \mu, \omega_{Z}\right) \mathrm{e}^{i\left[\left(\omega_{\nu}-\omega_{\mu}\right) t-\left(k_{\nu}-k_{\mu}\right) r\right]} \\
& B_{q}^{k}\left(v, \nu, \mu, \omega_{Z}\right)=-\sum_{q_{1} q_{2}}(-)^{J_{a}+J_{b}+q_{2}} N(v)\left|P_{a b}\right|^{2} \mathscr{E}_{q_{1}}^{\nu^{*}} \mathscr{E}_{-q_{2}}^{\mu}\left\langle 11 q_{1} q_{2} \mid k q\right\rangle\left\{\begin{array}{ccc}
k & 1 & 1 \\
J_{a} & J_{b} & J_{b}
\end{array}\right\} \\
& \times\left[\left[\Gamma_{a b}(1)+i\left(\omega+q_{2} \omega_{Z}-\omega_{\mu}+k_{\mu} v\right)\right]^{-1}+\left[\Gamma_{a b}(1)-i\left(\omega-q_{1} \omega_{Z}-\omega_{\nu}+k_{\nu} v\right)\right]^{-1}\right] .
\end{aligned}
$$

L'équation (I.30) devient :

$$
\begin{aligned}
{ }_{b}^{(2)} \dot{\rho}_{q}^{k} & =-\left(\Gamma_{b}(k)+i q \omega_{b}\right) \\
& +\sum_{\nu \mu} B_{q}^{k}\left(v, \nu, \mu, \omega_{Z}\right) \mathrm{e}^{i\left[\left(\omega_{\nu}-\omega_{\mu}\right) t^{\prime}-\left(k_{\nu}-k_{\mu}\right) r^{\prime}\right]} .
\end{aligned}
$$

En résolvant cette équation par rapport à $\tau$, comme nous l'avons fait pour l'équation (I.28), nous trouvons:

$$
\begin{aligned}
& \underset{b \rho_{q}^{k}(v, r, t)}{\left({ }^{(2)}\right)} \\
& \quad=\sum_{\nu \mu} \frac{B_{q}^{k}\left(v, \nu, \mu, \omega_{Z}\right) \mathrm{e}^{i\left[\left(\omega_{\nu}-\omega_{\mu}\right) t-\left(k_{\nu}-k_{\mu}\right) r\right]}}{\Gamma_{b}(k)+i\left(\omega_{\nu}-\omega_{\mu}+q \omega_{b}\right)} .
\end{aligned}
$$

Cette formule attire plusieurs remarques :

- Au dénominateur, nous avons négligé un terme en $\left(k_{v}-k_{\mu}\right) v$. Ce terme, correspondant à un effet Doppler à la fréquence $\omega_{\nu}-\omega_{\mu}$, est négligeable. En effet, $\omega_{\nu}-\omega_{\mu} \sim 2 \pi \times 10^{8}$ hertz $\sim 10^{-7} \omega_{\nu}$, donc $\left(k_{\nu}-k_{\mu}\right) v \sim 10^{-7} k_{\nu} v \sim 10^{-7} \times$ largeur Doppler $\sim 100$ hertz.

- La présence du coefficient $(6 \mathrm{~J})$ dans l'expression (I.32) de $B_{q}^{k}\left(v, \nu, \mu, \omega_{a}\right)$ montre que $k$ ne peut prendre que les valeurs 0,1 et 2 si $J_{a} \geqslant 1$ et la seule valeur $k=0$, si $J_{a}=0$.
- Nous voyons dès maintenant apparaître une modulation de $\rho$ à toutes les fréquences de battement entre modes. G'est ce qui se traduira par une modulation de la lumière de fluorescence [3].

Calculons maintenant ${ }_{a}^{(2)} \rho_{q}^{k}(v, r, t)$ :

$$
\begin{aligned}
& { }_{a}^{(2)} \rho_{q}^{k}=-\left(i q \omega_{a}+\Gamma_{a}(k)\right){ }_{a}^{(2)} \rho_{q}^{k} \\
& \quad+\Theta(b, a, k){ }_{b}^{(2)} \rho_{q}^{k}-i \operatorname{Tr}\left(\left[R,{ }^{(1)} \rho\right]_{a} T_{q}^{(k)+}\right) .
\end{aligned}
$$

L'équation différentielle linéaire (I.35) présente deux termes source, l'un provenant du laser, l'autre de l'émission spontanée. La solution stationnaire est la somme des solutions stationnaires correspondant à chacun des termes source. La solution correspondant au terme laser est analogue à la solution (I.34) de l'équation (I.30) : il suffit de remplacer $b$ par $a$ et $B_{q}^{k}$ par $A_{q}^{k}=(-1)^{k+1} B_{q}^{k} \frac{\left\{\begin{array}{ccc}k & 1 & 1 \\ J_{b} & J_{a} & J_{a}\end{array}\right\}}{\left\{\begin{array}{ccc}k & 1 & 1 \\ J_{a} & J_{\iota} & J_{\iota}\end{array}\right\}}$. 
On trouve :

$$
\begin{aligned}
{ }_{a}^{(2)} \rho_{q}^{k}(v, r, t) & =\sum_{\nu \mu} \frac{A_{q}^{k}\left(v, \nu, \mu, \omega_{Z}\right) \mathrm{e}^{i\left[\left(\omega_{\nu}-\omega_{\mu}\right) t-\left(k_{\nu}-k_{\mu}\right) r\right]}}{\Gamma_{\mathrm{a}}(k)+i\left(\omega_{\nu}-\omega_{\mu}+\mathrm{q} \omega_{\mathrm{a}}\right)}+\sum_{\mu \nu} \frac{B_{q}^{k}\left(v, \nu, \mu, \omega_{Z}\right) \Theta(b, a, k) \mathrm{e}^{i\left[\left(\omega_{\nu}-\omega_{\mu}\right) t-\left(k_{\nu}-k_{\mu}\right) r\right]}}{\left[\Gamma_{a}(k)+i\left(\omega_{\nu}-\omega_{\mu}+q \omega_{a}\right)\right]\left[\Gamma_{b}(k)+i\left(\omega_{\nu}-\omega_{\mu}+q \omega_{b}\right)\right]} \\
& =\sum_{\nu \mu} \frac{A_{\mathrm{q}}^{\mathrm{k}}\left(v, \nu, \mu, \omega_{\mathrm{Z}}\right) \mathrm{e}^{i[\ldots]}}{\Gamma_{\mathrm{a}}(k)+i\left(\omega_{\nu}-\omega_{\mu}+\mathrm{q} \omega_{\mathrm{a}}\right)}\left[1-(-)^{k} \frac{\left\{\begin{array}{lll}
k & 1 & 1 \\
J_{a} & J_{b} & J_{b}
\end{array}\right\}}{\left\{\begin{array}{lll}
k & 1 & 1 \\
J_{b} & J_{a} & J_{a}
\end{array}\right\}} \frac{\Theta(b, a, k)}{\Gamma_{b}(k)+i\left(\omega_{\nu}-\omega_{\mu}+q \omega_{b}\right)}\right] . \quad \text { (I.36) }
\end{aligned}
$$

5. INTÉGration SUR LeS VITESSES. $-A_{q}^{k}\left(v, \nu, \mu, \omega_{Z}\right)$ et $B_{q}^{k}\left(v, \nu, \mu, \omega_{Z}\right)$ dépendent de la vitesse des atomes par des termes de la forme :



Nous supposerons tous les $k_{\nu}$ égaux et nous poserons:

$$
\begin{array}{r}
N(\Omega)=\int_{-\infty}^{+\infty} \frac{N(v) \mathrm{d} v}{\Gamma_{a b}-i(\Omega+k v)} \\
N(v)=\left(\frac{\lambda_{b}}{\Gamma_{b}^{(0)}}-\frac{\lambda_{a}}{\Gamma_{a}^{(0)}}\right) \frac{1}{u \sqrt{\pi}} \mathrm{e}^{-(v / u)^{2}}=n \frac{k}{\Delta \nu \sqrt{\pi}} \mathrm{e}^{-(k v / \Delta v)^{2}} .
\end{array}
$$

En posant :

$\Delta \nu=k u=k \sqrt{2 R T / M}=\Delta \nu_{\mathrm{D}} / 2 \sqrt{\log 2}$

$T=$ température du gaz,

$M=$ masse molaire $=20 \mathrm{~g}$ pour le néon,

$R=$ constante des gaz parfaits,

$\Delta v_{\mathrm{D}}=$ largeur Doppler de la raie [14], avec $x=-k v / \Delta \nu$, on obtient :

$$
\begin{aligned}
N(\Omega)=-\frac{i n}{\Delta \nu \sqrt{\pi}} \int_{-\infty}^{+\infty} \frac{\mathrm{e}^{-x^{2}} \mathrm{~d} x}{x-\frac{\Omega+i \Gamma_{a b}}{\Delta \nu}} \\
=-\frac{i n}{\Delta \nu} Z\left(\frac{\Omega}{\Delta \nu}+i \frac{\Gamma_{a b}}{\Delta \nu}\right)
\end{aligned}
$$

$Z$ est la fonction de dispersion des plasmas et ses valeurs sont tabulées [15].

Enfin, nous poserons :

$$
\begin{gathered}
N(\Omega)=\frac{n}{\Delta \nu}\left[X\left(\frac{\Omega}{\Delta \nu}\right)+i Y\left(\frac{\Omega}{\Delta \nu}\right)\right] \\
X \text { et } Y \text { sont réels } \\
X\left(\frac{\Omega}{\Delta \nu}\right)=\operatorname{Im}\left[Z\left(\frac{\Omega}{\Delta \nu}+i \frac{\Gamma_{a b}}{\Delta \nu}\right)\right] \\
\text { est une fonction paire de } \Omega \\
Y\left(\frac{\Omega}{\Delta \nu}\right)=-\operatorname{Re}\left[Z\left(\frac{\Omega}{\Delta \nu}+i \frac{\Gamma_{a b}}{\Delta \nu}\right)\right] \\
\text { est une fonction impaire de } \Omega . \quad(\mathrm{I} .41)
\end{gathered}
$$

En général, $\Gamma_{a b}$ est petit devant $\Delta v$. Il en résulte que $X(\Omega)$ est peu différent d'une gaussienne $\mathrm{e}^{-\Omega^{2} / \Delta v}$ et que $N(\Omega)$ dépend peu de la valeur de $\Gamma_{a b}$. Pour cette raison, l'étude expérimentale des effets linéaires par rapport à l'intensité du laser (ordre (2)) est pratiquement insensible à la relaxation des éléments non diagonaux « optiques »:

$$
\begin{aligned}
{ }_{b}^{(2)} \rho_{q}^{k}(r, t)=-\left|P_{a b}\right|^{2} \sum_{\nu \mu} & \frac{\mathrm{e}^{i\left[\left(\omega_{\nu}-\omega_{\mu}\right) t-\left(k_{\nu}-k_{\mu}\right) r\right]}}{\Gamma_{b}(k)+i\left(\omega_{\nu}-\omega_{\mu}+q \omega_{b}\right)} \sum_{q_{1} q_{2}} \mathscr{E}_{q_{1}} \mathscr{E}_{q_{2}}^{\mu}\left\langle 11 q_{1}-q_{2} \mid k q\right\rangle \\
& \times(-)^{J_{a}+J_{b}+q_{2}}\left\{\begin{array}{ccc}
k & 1 & 1 \\
J_{a} & J_{b} & J_{b}
\end{array}\right\}\left[N\left(\omega-q_{1} \omega_{Z}-\omega_{\mu}\right)+N^{*}\left(\omega-q_{2} \omega_{Z}-\omega_{\mu}\right)\right] .
\end{aligned}
$$

${ }_{b}^{(2)} \rho_{q}^{k}(r, t)$ dépend du champ magnétique de deux façons différentes :

1) Par les termes $\frac{1}{\Gamma_{b}(k)+i\left(\omega_{\nu}-\omega_{\mu}+q \omega_{b}\right)}$ qui sont résonnants (si $q \neq 0$ ) lorsque la fréquence de précession $q \omega_{b}$ du terme ${ }_{b} \rho_{q}^{k}$ est égale à la fréquence d'oscillation forcée $\omega_{\nu}-\omega_{\mu}$ imposée par les modes $\mathrm{du}$ laser. Ces termes présentent une résonance de largeur $\Gamma_{b}(k)$ (de l'ordre d'une dizaine de $\mathrm{MHz}$ ).

2) Par les termes de la forme $N\left(\omega-q \omega_{Z}-\omega_{v}\right)$. Ces termes traduisent la forme Doppler de la raie. La largeur de la courbe correspondante est de l'ordre de $\Delta v$, c'est-à-dire environ $1000 \mathrm{MHz}$ pour une raie visible. 
Comme $\Delta \nu \gg \Gamma_{b}(k)$, on peut admettre que les fonctions $N\left(\omega-q \omega_{z}-\omega_{v}\right)$ varient peu lorsque l'on fait varier le champ magnétique de façon à balayer la résonance $\frac{1}{\Gamma_{b}(k)+i\left(\omega_{\nu}-\omega_{\mu}+q \omega_{b}\right)}$. On peut donc considérer que ce dernier facteur détermine la forme de la résonance, les facteurs $N(\omega)$ ne jouant que sur l'amplitude.
6. L'effet Hanle. - Dans la sommation sur $\nu$ et $\mu$, nous trouvons des termes de fréquence nulle. Ce sont les seuls termes auxquels nous serons sensibles si nous mesurons la lumière de fluorescence avec un appareillage muni d'une forte constante de temps.

Ces termes « continus » $q \neq 0$ sont résonnants pour $H=0$ : c'est l'effet Hanle. Explicitons ces termes :

$$
\begin{aligned}
& { }_{b}^{(2)} \rho_{q}^{k}(\text { continu })=-\left|P_{a b}\right|^{2} \frac{1}{\Gamma_{b}(k)+i q \omega_{b}} \sum_{\mu q_{1} q_{2}} \mathscr{E}_{q_{1}}^{\mu^{*}} \mathscr{E}_{q_{2}}^{\mu}(-)^{J_{a}+J_{b}+q_{2}}\left\langle 11 q_{1}-q_{2} \mid k q\right\rangle \\
& \left\{\begin{array}{ccc}
k & 1 & 1 \\
J_{a} & J_{b} & J_{b}
\end{array}\right\}\left[N\left(\omega-q_{1} \omega_{Z}-\omega_{\mu}\right)+N^{*}\left(\omega-q_{2} \omega_{Z}-\omega_{\mu}\right)\right] \\
& { }_{a}^{(2)} \rho_{q}^{k}(\text { continu })=+\left|P_{a b}\right|^{2} \frac{1}{\Gamma_{a}(k)+i q \omega_{a}}\left[1-(-)^{k} \frac{\left\{\begin{array}{ccc}
k & 1 & 1 \\
J_{a} & J_{b} & J_{b}
\end{array}\right\}}{\left\{\begin{array}{lll}
k & 1 & 1 \\
J_{b} & J_{a} & J_{a}
\end{array}\right\}} \frac{\Theta(b, a, k)}{\Gamma_{b}(k)+i q \omega_{b}}\right] . \\
& \sum_{\mu q_{1} q_{2}} \mathscr{E}_{q_{1}}^{\mu^{*}} \mathscr{E}_{q_{2}}^{\mu}(-)^{J_{a}+J_{b+q_{2}}}\left\langle 11-q_{2} q_{1} \mid k q\right\rangle\left\{\begin{array}{ccc}
k & 1 & 1 \\
J_{b} & J_{a} & J_{a}
\end{array}\right\}\left[N\left(\omega-q_{1} \omega_{Z}-\omega_{\mu}\right)+N^{*}\left(\omega-q_{2} \omega_{Z}-\omega_{\mu}\right)\right] .
\end{aligned}
$$

a) Pour $q=0 \quad\left(q_{1}=q_{2}\right)$, nous n'avons pas de premier facteur d'allure résonnante. La seule variation est due aux termes Doppler :

$$
\begin{aligned}
& { }_{b}^{(2)} \rho_{0}^{k}=-\frac{\left|P_{a b}\right|^{2}}{\Gamma_{b}(k)} \sum_{\mu q_{1}}\left|\mathscr{E}_{q_{1}}^{\mu}\right|^{2}\left\langle 11 q_{1}-q_{1} \mid k 0\right\rangle(-)^{J_{a}+J_{b}+q_{1}} \\
& \frac{2 n}{\Delta \nu} X\left(\frac{\omega-\omega_{\mu}-q_{1} \omega_{Z}}{\Delta \nu}\right)
\end{aligned}
$$

$X\left(\frac{\omega-\omega_{\mu}-q_{1} \omega_{Z}}{\Delta \nu}\right)$ reproduit simplement la forme Doppler ordinaire.

b) Pour $q \neq 0$, nous pouvons négliger la variation de $N\left(\omega-q_{1} \omega_{Z}-\omega_{\mu}\right)$ dans la région où :

$$
1 /\left(\Gamma_{\alpha}(k)-i q \omega_{\alpha}\right)
$$

a une valeur importante et remplacer :

$$
N\left(\omega-q_{1} \omega_{Z}-\omega_{\mu}\right)+N^{*}\left(\omega-q_{2} \omega_{Z}-\omega_{\mu}\right)
$$

par :

$$
2 \frac{n}{\Delta \nu} X\left(\frac{\omega-\omega_{\mu}}{\Delta \nu}\right)
$$

Dans certains cas, il sera préférable de remplacer cette expression par :

$\frac{n}{\Delta \nu}\left[X\left(\frac{\omega-q_{1} \omega_{Z}-\omega_{\mu}}{\Delta \nu}\right)+X\left(\frac{\omega-q_{2} \omega_{Z}-\omega_{\mu}}{\Delta \nu}\right)\right]$ ce qui ne change rien tant que $\omega_{Z} \ll \Delta \nu$.
Remarques. - Nous retrouvons des résultats bien connus. On ne peut pas obtenir des grandeurs transversales $(q \neq 0)$ si on excite avec une onde de polarisation pure $\sigma^{+}, \sigma^{-}$ou $\pi\left(q_{1}=q_{2} \rightarrow q=0\right)$.

- Si le laser est de polarisation plane, les symétries de (I.43) et (I.44) montrent que l'on ne peut créer d'orientation $(k=1)$ que si le champ magnétique est suffisant pour séparer sensiblement les formes Doppler $\sigma^{+}, \sigma^{-}$et $\pi$. Dans l'approximation faite ici des grandes largeurs Doppler, nous pouvons négliger l'orientation.

- Nous trouvons ici la justification de l'hypothèse faite au § C. 1 sur la structure Zeeman. Si nous avions fait le calcul complet en conservant les termes de couplage en $\omega_{b}-\omega_{a}$ des équations (I.25c), cette différence $\omega_{b}-\omega_{a}$ n'interviendrait dans (I.43) que par des termes de la forme :

$N\left(\omega-\omega_{\mu}+M_{b} \omega_{b}-M_{a} \omega_{a}\right)$

$=N\left(\omega-\omega_{\mu}-q_{1} \omega_{Z}+\frac{1}{2}\left(M_{b}+M_{a}\right)\left(\omega_{b}-\omega_{a}\right)\right)$

puisque $q_{1} \omega_{Z}$ est négligeable devant $\Delta \nu, M\left(\omega_{b}-\omega_{a}\right)$ l'est a fortiori.

Seule la formule (I.45) risque d'être modifiée en champ très fort, mais il est évident qu'au lieu d'être la superposition des trois formes Doppler $\sigma^{+}, \sigma^{-}$et $\pi$, elle sera la superposition d'autant de formes Doppler qu'il y a de composantes Zeeman. 
- Si tous les modes ont la même polarisation, nous pouvons poser $\mathscr{E}^{\mu}=\mathbf{e} \mathscr{E}^{\mu}$ où $\mathbf{\theta}$ est le vecteur unitaire polarisation. On peut alors écrire :

$$
\begin{aligned}
&{ }_{b}^{(2)} \rho_{q}^{k}=-\left|P_{a b}\right|^{2} \bar{\Gamma}_{b}(k)+i q \omega_{b} \sum \mathrm{q}_{1} q_{2} \\
& \mathrm{e}_{q_{1}}^{*} \mathrm{e}_{q_{2}}\left\langle 11 q_{1}-q_{2} \mid k q\right\rangle\left\{\begin{array}{ccc}
k & 1 & 1 \\
J_{a} & J_{b} & J_{b}
\end{array}\right\} \\
& \times(-)^{J_{a}+J_{b}+q_{2}} \sum_{\mu}\left|\mathscr{E}^{\mu}\right|^{2}\left[N\left(\omega-q_{1} \omega_{Z}-\omega_{\mu}\right)+N^{*}\left(\omega-q_{2} \omega_{Z}-\omega_{\mu}\right)\right]
\end{aligned}
$$

$\left|\mathscr{E}^{\mu}\right|^{2}$ est proportionnel à l'intensité $I_{\mu}$ du mode $\mu$.

On voit que ${ }^{(2)} \rho_{q}^{k}$ n'est pas proportionnel à l'intensité totale du laser car les modes loin du centre de la raie sont moins efficaces. Lorsque l'on fait varier la puissance du laser en modifiant les pertes de la cavité, le nombre de modes varie. Ceci produit une cause de non-linéarité dans l'étude de la variation de la lumière de fluorescence en fonction de l'intensité totale du laser. Ceci est à distinguer des phénomènes de saturation liés aux termes en $I^{2}, I^{3}, \ldots$, etc. Par contre, les variations de la largeur de l'effet Hanle en fonction de $I$ ne peuvent provenir que des saturations.

7. Cas particulier d'une onde laser se propageant parallèlement à $\mathrm{Oz}$ et polarisée parallèlement à $\mathrm{Ox}$ :

$$
\mathscr{E}^{\mu}=\mathscr{E}^{\mu} \mathbf{e}_{x} ; \quad \mathscr{E}_{ \pm 1}^{\mu}=\mp \frac{1}{\sqrt{2}} \mathscr{E}^{\mu} .
$$

On a alors :

$$
\begin{aligned}
& { }_{b \rho_{0}^{0}}^{(2)}=-\frac{\left|P_{a b}\right|^{2}}{\Gamma_{b}(0)} \frac{1}{3 \sqrt{2 J_{b}+1}} D\left(\omega_{Z}\right) \\
& { }_{b}^{(2)} \rho_{0}^{2}=-\frac{\left|P_{a b}\right|^{2}}{\Gamma_{b}(2)} \frac{(-)^{J_{a}+J_{b}+1}}{\sqrt{\overline{6}}}\left\{\begin{array}{ccc}
2 & 1 & 1 \\
J_{a} & J_{b} & J_{b}
\end{array}\right\} D\left(\omega_{Z}\right) \\
& { }_{b}^{(2)} \rho_{ \pm 2}^{2}=\frac{\left|P_{a b}\right|^{2}}{2} \frac{(-)^{J_{a}+J_{b}+1}}{\Gamma_{b}(2) \pm 2 i \omega_{b}}\left\{\begin{array}{ccc}
2 & 1 & 1 \\
J_{a} & J_{b} & J_{b}
\end{array}\right\} D\left(\omega_{Z}\right) \\
& { }_{a}^{(2)} \rho_{0}^{0}=\frac{\left|P_{a b}\right|^{2}}{\Gamma_{a}(0)} \frac{1}{3 \sqrt{2 J_{a}+1}}\left[1-\frac{\gamma_{b a}}{\Gamma_{b}(0)}\right] D\left(\omega_{Z}\right) \\
& { }_{a}^{(2)} \rho_{0}^{2}=\frac{\left|P_{a b}\right|^{2}}{\Gamma_{a}(2)} \frac{(-)^{J_{a}+J_{b}+1}}{\sqrt{6}}\left\{\begin{array}{ccc}
2 & 1 & 1 \\
J_{b} & J_{a} & J_{a}
\end{array}\right\}\left[1-(-)^{J_{a}+J_{b}+1} \frac{\gamma_{b a}}{\Gamma_{b}(2)}\left(2 J_{b}+1\right) \frac{\left\{\begin{array}{ccc}
2 & 1 & 1 \\
J_{a} & J_{b} & J_{b}
\end{array}\right\}\left\{\begin{array}{lll}
2 & J_{b} & J_{b} \\
1 & J_{a} & J_{a}
\end{array}\right\}}{\left\{\begin{array}{ccc}
2 & 1 & 1 \\
J_{b} & J_{a} & J_{a}
\end{array}\right\}}\right] D\left(\omega_{Z}\right) \\
& \stackrel{(2) \rho_{ \pm 2}^{2}}{2}=-\left|P_{a b}\right|^{2} \frac{(-)^{J_{a}+J_{b}+1}}{\Gamma_{a}(2) \pm 2 i \omega_{a}}\left\{\begin{array}{ccc}
2 & 1 & 1 \\
J_{b} & J_{a} & J_{a}
\end{array}\right\}\left[1-(-)^{J_{a}+J_{b}+1} \frac{\gamma_{b a}\left(2 J_{b}+1\right)}{\Gamma_{b}(2) \pm 2 i \omega_{b}} \frac{\left\{\begin{array}{ccc}
2 & 1 & 1 \\
J_{a} & J_{b} & J_{b}
\end{array}\right\}\left\{\begin{array}{lll}
2 & J_{b} & J_{b} \\
1 & J_{a} & J_{a}
\end{array}\right\}}{\left\{\begin{array}{ccc}
2 & 1 & 1 \\
J_{b} & J_{a} & J_{a}
\end{array}\right\}}\right] D\left(\omega_{Z}\right) .
\end{aligned}
$$

où :

$$
\begin{aligned}
D\left(\omega_{Z}\right)=\frac{n}{\Delta \nu} \sum_{\mu}\left|\mathscr{E}^{\mu}\right|^{2}\left[X\left(\frac{\omega-\omega_{\mu}+\omega_{Z}}{\Delta \nu}\right)\right. \\
\left.+X\left(\frac{\omega-\omega_{\mu}-\omega_{Z}}{\Delta \nu}\right)\right]
\end{aligned}
$$

contient l'effet Doppler et l'influence de la structure des modes du laser.

D. Emissions de lumière de fluorescence par les niveaux $a$ ou $b$. - Nous poserons les équations pour la raie $b \rightarrow g$. Pour la raie $a \rightarrow f$, tout est identique dans ce paragraphe, au remplacement près de $b$ par $a$ et $g$ par $f$.

La lumière de fluorescence émise dans un angle solide $\Delta \Omega$ avec la polarisation $\lambda$ s'écrit (CohenTannoudji [13], formule (III.B.1) généralisée pour les vecteurs $\boldsymbol{\theta}_{\lambda}$ complexes) :

$$
\begin{aligned}
L_{F, b g}(\boldsymbol{\lambda}) & =\frac{3 \gamma_{b g}}{8 \pi} \sum_{M_{b} M_{b}^{\prime}} \rho_{M_{b} M_{b}^{\prime}}\left\langle M_{b}^{\prime}|\boldsymbol{\lambda} \mathbf{D}| M_{g}\right\rangle \\
& \left\langle M_{g}\left|\boldsymbol{\lambda}^{*} \mathbf{D}\right| M_{b}\right\rangle \\
& =\frac{3 \gamma_{b g}}{8 \pi} \operatorname{Tr}\left[{ }_{b} \rho(\boldsymbol{\lambda} \mathbf{D})\left(\boldsymbol{\lambda}^{*} \mathbf{D}\right)\right]
\end{aligned}
$$


où $\gamma_{b g}$ est la probabilité de transition $b \rightarrow g$ par unité de temps.

D est un opérateur vectoriel hermitique proportionnel à $P$ et n'ayant d'éléments de matrice qu'entre $b$ et $g$, tel que :

$$
\left\langle J_{b} M_{b}\left|D_{q}\right| J_{g} M_{g}\right\rangle=\left\langle J_{g} 1 M_{g} q \mid J_{b} M_{b}\right\rangle
$$

ce qui donne :

$$
\langle b\|D\| g\rangle=(-1)^{J_{g}-J_{b}}\langle g\|\mathbf{D}\| b\rangle=\sqrt{2 J_{b}+1} .
$$

Nous pouvons exprimer $\boldsymbol{\lambda}$ et $\mathbf{D}$ en fonction de leurs composantes standard et décomposer $\rho$ en opérateurs tensoriels irréductibles :

$$
\begin{aligned}
L_{F, b g}(\boldsymbol{\lambda}) & =\frac{3 \gamma_{b g}}{8 \pi} \sum_{k q p p^{\prime}}{ }_{b} \rho_{q}^{k}(r, t) \operatorname{Tr}\left[{ }_{b} T_{q}^{(k)}\left((-)^{p} \lambda_{-p} D_{p}\right)\left((-)^{p^{\prime}}\left(\lambda^{*}\right)_{-p^{\prime}} D_{p^{\prime}}\right)\right] \\
& =\frac{3 \gamma_{b g}}{8 \pi} \sum_{k q p p^{\prime}}(-)^{J_{b}+J_{g^{+}} p^{\prime}}{ }_{b \rho_{q}}^{k}(r, t) \lambda_{p} \lambda_{p^{\prime}}^{*}\left(2 J_{b}+1\right) \cdot\left\langle 11 p-p^{\prime} \mid k q\right\rangle\left\{\begin{array}{ccc}
k & 1 & 1 \\
J_{g} & J_{b} & J_{b}
\end{array}\right\}
\end{aligned}
$$

Cette formule permet de calculer directement le diagramme de rayonnement de la lumière de fluorescence dès que l'on connaît les éléments ${ }_{b} \rho_{q}^{k}$.

Il nous reste à donner l'expression des composantes $\lambda_{p}$ pour une direction d'observation et une polarisation quelconque (fig. 2). La direction d'obser-

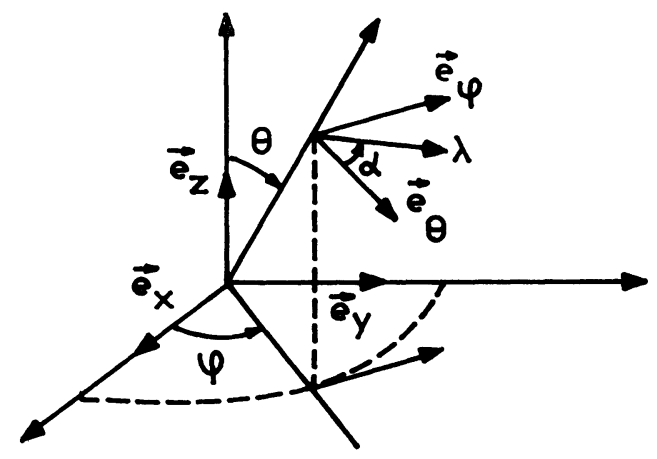

FIG. 2 .

vation est définie par les angles $\theta$ et $\varphi$. La polarisation est repérée par rapport aux deux vecteurs $\mathbf{e}_{\theta}$ et $\mathbf{e}_{\varphi}\left(\mathbf{e}_{\varphi}\right.$ parallèle à $\left.\mathrm{xOy}\right)$ du plan normal à la direction d'observation.

Polarisation plane :

$$
\begin{aligned}
\boldsymbol{\lambda} & =\mathbf{e}_{\theta} \cos \alpha+\mathbf{e}_{\varphi} \sin \alpha \\
\lambda_{0} & =-\cos \alpha \sin \theta \\
\lambda_{ \pm 1} & =\mp(1 / \sqrt{2})(\cos \alpha \cos \theta \pm i \sin \alpha) \mathrm{e}^{ \pm i \varphi} .
\end{aligned}
$$

Polarisation circulaire :

$$
\begin{aligned}
\lambda^{\mathrm{d}} & =-(1 / \sqrt{2})\left(\mathbf{e}_{\theta}+i \mathbf{e}_{\varphi}\right) \\
\lambda_{0}^{d} & =(1 / \sqrt{2}) \sin \theta \\
\lambda_{ \pm 1}^{d} & = \pm \frac{\cos \theta \mp 1}{2} \mathrm{e}^{ \pm i \varphi} \\
\lambda^{\mathrm{g}} & =+(1 / \sqrt{2})\left(\mathbf{e}_{\theta}-i \mathbf{e}_{\varphi}\right) \\
\lambda_{0}^{g} & =-(1 / \sqrt{2}) \sin \theta \\
\lambda_{ \pm 1}^{g} & =\mp \frac{\cos \theta \pm 1}{2} \mathrm{e}^{ \pm i \varphi} .
\end{aligned}
$$

E. Définition de grandeurs observables expérimentalement. - Dans ce paragraphe, nous nous proposons de définir plusieurs grandeurs mesurables et de montrer comment elles sont reliées aux divers temps de relaxation.

Nous nous plaçons dans le cas particulier du $\S \mathrm{C} .7$ (formules (I.47)) : l'onde laser se propage parallèlement à $\mathrm{Oz}$ (axe du champ magnétique) et elle est polarisée parallèlement à $\mathrm{Ox}$.

Nous considérons les directions d'observation perpendiculaires à $\mathrm{Oz}(\theta=\pi / 2)$. Pour chaque direction repérée par $\varphi$ dans le plan xOy (fig. 2), nous mesurons l'intensité des deux polarisations planes $\pi$ et $\sigma$ de chacune des raies de fluorescence issues du niveau $a$ ou $b$. Pour le niveau supérieur $b$, la formule (I.51) donne (pour la polarisation $\pi: \lambda_{0}=1, \lambda_{ \pm 1}=0$, et pour la polarisation $\left.\sigma: \lambda_{0}=0 ; \lambda_{ \pm}=-(i / \sqrt{2}) \mathrm{e}^{ \pm i \varphi}\right)$ :

$$
\begin{aligned}
& L_{F, b g}^{\pi}=\frac{3 \gamma_{b q}\left(1-x_{b g}\right)}{8 \pi}\left(2 J_{b}+1\right)\left[\frac{1}{3 \sqrt{2 J_{b}+1}} b \rho_{0}^{0}+\sqrt{\frac{2}{3}}{ }_{b} \rho_{0}^{2}(-)^{J_{b}+J_{g}}\left\{\begin{array}{ccc}
2 & 1 & 1 \\
J_{g} & J_{b} & J_{b}
\end{array}\right)\right] \\
& L_{F, b g}^{\sigma}=\frac{3 \gamma_{b q}\left(1-x_{b g}\right)}{8 \pi}\left(2 J_{b}+1\right)\left[\frac{1}{3 \sqrt{2 J_{b}+1}} b \rho_{0}^{0}+\frac{1}{\sqrt{6}} b \rho_{0}^{2}(-)^{J_{b}+J_{g}+1}\left\{\begin{array}{ccc}
2 & 1 & 1 \\
J_{g} & J_{b} & J_{b}
\end{array}\right\}\right. \\
& \left.+\frac{1}{2}\left({ }_{b} \rho_{2}^{2} \mathrm{e}^{2 i \varphi}+{ }_{b} \rho_{-2}^{2} \mathrm{e}^{-2 i \varphi}\right)(-)^{J_{b}+J_{g}+1}\left\{\begin{array}{ccc}
2 & 1 & 1 \\
J_{g} & J_{b} & J_{b}
\end{array}\right\}\right] \text {. }
\end{aligned}
$$


Comme avec toute polarisation plane, on ne peut pas observer les composantes de l'orientation $(k=1)$.

Dans les formules (I.52), on peut utiliser la décomposition de la matrice densité en ordres successifs :

$L_{F}={ }^{(0)} L_{F}\left\{{ }^{(0)} \rho\right\}+{ }^{(2)} L_{F}\left\{{ }^{(2)} \rho\right\}+{ }^{(4)} L_{F}\left\{{ }^{(4)} \rho\right\}+\ldots$

${ }^{(0)} L_{F}$ est l'intensité de la lumière de fluorescence; en l'absence d'irradiation laser, elle ne dépend que de la population. La variation de $L_{F}$ sous l'effet du laser est :

$$
\Delta L_{F}={ }^{(2)} L_{F}+{ }^{(4)} L_{F}+\ldots
$$

Si l'intensité du laser $i_{\lambda}$ est très faible, $\Delta L_{F}$ est sensiblement égal à ${ }^{(2)} L_{F}$. ${ }^{(2)} L_{F, b g}^{\pi}$ et ${ }^{(2)} L_{F, b g}^{\sigma}$ sont des combinaisons linéaires de ${ }_{b}^{(2)} \rho_{0}^{0}$ (variation de population), ${ }_{b}^{(2)} \rho_{0}^{2}$ et ${ }_{b}^{(2)} \rho_{ \pm 2}^{2} \quad$ (alignements longitudinal et transversal). La détermination expérimentale de ${ }^{(2)} L_{F}^{\pi}$ et de ${ }^{(2)} L_{F}^{\sigma}$ pour les différentes raies de fluorescence issues de $b$ nous permet donc de calculer la valeur relative de la population et des alignements, ainsi que leur temps de relaxation.

1. Étude de la polarisation $\pi$. Définition de $F$. - $L_{F}^{\pi}$ ne dépend pas de l'angle $\varphi$ d'observation. D'autre part, ${ }^{(2)} L_{F}^{\pi}$ ne dépend que de ${ }_{b}^{(2)} \rho_{0}^{0}$ et ${ }_{b}^{(2)} \rho_{0}^{2}$ qui sont indépendants du champ magnétique (tant que $\left.\omega_{Z} \ll \Delta \nu\right)$. La comparaison de deux raies de fluorescence $b \rightarrow g$ et $b \rightarrow g^{\prime}$, telles que $J_{g} \neq J_{g^{\prime}}$, permet d'obtenir le rapport ${ }_{b}^{(2)} \rho_{0}^{0} /(2) \rho_{0}^{2}$.

Pour cela, nous définissons la grandeur :

$$
F\left(b g g^{\prime}\right)=\frac{\Delta L_{F, b g}^{\pi}}{\Delta L_{F, b g^{\prime}}^{\pi}} \frac{L_{F, b g^{\prime}}^{\pi}}{L_{F, b g}^{\pi}} .
$$

En utilisant (I.53), on définit :

$$
F^{(1)}\left(b g g^{\prime}\right)=\operatorname{limite}_{i \lambda \rightarrow 0} F\left(b g g^{\prime}\right)=\operatorname{limite}_{i \lambda \rightarrow 0} \frac{{ }^{(2)} L_{F, b g}^{\pi}}{{ }^{(2)} L_{F, b g^{\prime}}^{\pi}} \frac{{ }^{(0)} L_{F, b g^{\prime}}^{\pi}}{{ }^{(0)} L_{F, b g}^{\pi}} .
$$

Les formules (I.52) et (I.47) permettent d'écrire :

$$
\begin{aligned}
& F^{(1)}\left(b g g^{\prime}\right)=\frac{1+(-)^{J_{b}+J_{g}} \sqrt{6\left(2 J_{b}+1\right)}\left\{\begin{array}{ccc}
2 & 1 & 1 \\
J_{g} & J_{b} & J_{b}
\end{array} \frac{{ }_{b}^{(2)} \rho_{0}^{2}}{(2) \rho_{0}^{0}}\right.}{1+(-)^{J_{b}+J_{g^{\prime}}} \sqrt{6\left(2 J_{b}+1\right)}\left\{\begin{array}{ccc}
2 & 1 & 1 \\
J_{g^{\prime}} & J_{b} & J_{b}
\end{array}\right\} \frac{(2) \rho_{0}^{(2)}}{(2) \rho_{0}^{0}}} \\
& F^{(1)}\left(b g g^{\prime}\right)=\frac{1+(-)^{2 J_{b}+J_{a}+J_{g}+1}\left[3\left(2 J_{b}+1\right)\right]\left\{\begin{array}{ccc}
2 & 1 & 1 \\
J_{a} & J_{b} & J_{b}
\end{array}\right\}\left\{\begin{array}{ccc}
2 & 1 & 1 \\
J_{g} & J_{b} & J_{b}
\end{array}\right\} \frac{\Gamma_{b}(0)}{\Gamma_{b}(2)}}{1+(-)^{2 J_{b}+J_{a}+J_{g^{\prime}+1}}\left[3\left(2 J_{b}+1\right)\right]\left\{\begin{array}{ccc}
2 & 1 & 1 \\
J_{a} & J_{b} & J_{b}
\end{array}\right\}\left\{\begin{array}{ccc}
2 & 1 & 1 \\
J_{g^{\prime}} & J_{b} & J_{b}
\end{array}\right\} \frac{\Gamma_{b}(0)}{\Gamma_{b}(2)}} .
\end{aligned}
$$

Ainsi, nous obtenons autant de déterminations indépendantes du rapport $\Gamma_{b}(2) / \Gamma_{b}(0)$ que nous avons de couples de raies de fluorescence telles que $J_{g} \neq J_{g^{\prime}}$.

Remarquons que $F$ peut dépendre du champ magnétique par les termes de saturation [5] ${ }_{b}^{(4)} \rho$, mais que $F^{(1)}$ n'en dépend pas (tant que $\omega_{Z} \ll \Delta v$ ).

2. Étude de la polarisation $\sigma$ : efFet Hanle. DÉfinition DE $\Delta H$ ET $R$. - La formule (I.52) montre que $L_{F}^{\sigma}$ contient deux parties de comportement différent :

a) Une partie isotrope, c'est-à-dire indépendante de l'angle $\varphi$ de la direction d'observation (dans le plan $\mathrm{xOy}$ ) :

$$
\begin{aligned}
& \left.L_{F, b g}^{\sigma} \text { (iso }\right)=\frac{3 \gamma_{b g}\left(1-x_{b g}\right)}{8 \pi}\left(2 J_{b}+1\right) \\
& {\left[\frac{1}{3 \sqrt{2 J_{b}+1}}{ }_{b} \rho_{0}^{0}+\frac{1}{\sqrt{6}}{ }_{b} \rho_{0}^{2}(-)^{J_{b}+J_{g}+1}\left\{\begin{array}{ccc}
2 & 1 & 1 \\
J_{g} & J_{b} & J_{b}
\end{array}\right\}\right] .}
\end{aligned}
$$

Elle dépend seulement de la population et de l'alignement longitudinal.

b) Une partie anisotrope, qui dépend de $\varphi$ : c'est le terme en $\left({ }_{b} \rho_{2}^{2} \mathrm{e}^{\mathrm{i} \varphi}+{ }_{b} \rho_{-2}^{2} \mathrm{e}^{-i \varphi}\right)$.

Pour deux directions d'observation perpendiculaires, ce terme se traduit par une variation de la lumière égale et de signe contraire.

Expérimentalement, nous ne nous intéressons qu'aux angles d'observation $\varphi=0$ et $\varphi=\pi / 2$ (parallèle et perpendiculaire au plan de polarisation du laser). Pour ces deux directions, nous définirons :

$L_{F}^{\sigma}($ aniso $)=L_{F / l}^{\sigma}($ aniso $)=-L_{F \perp}^{\sigma}($ aniso $)=\frac{3 \gamma_{b g}\left(1-x_{b g}\right)}{8 \pi}\left(2 J_{b}+1\right) \operatorname{Re}\left({ }_{b} \rho_{2}^{2}\right)\left\{\begin{array}{ccc}2 & 1 & 1 \\ J_{g} & J_{b} & J_{b}\end{array}\right\}(-)^{J_{b}+J_{g}+1}$. 
De même que nous l'avons montré à l'ordre (2), on peut montrer qu'à tous les ordres ${ }_{b} \rho_{ \pm 2}^{2}$ s'annule dès que l'écart Zeeman est très supérieur à $\Gamma_{b}(2)$.

Cette variation anisotrope de la lumière de fluorescence, qui peut être détruite par un champ magnétique, constitue l'effet Hanle.

Avec les définitions (I.59) et (I.60), nous pouvons écrire :

$$
\left\{\begin{array}{l}
L_{F \prime l}^{\sigma}=L_{F}^{\sigma}(\text { iso })+L_{F}^{\sigma}(\text { aniso }) \\
\left.L_{F \perp}^{\sigma}=L_{F}^{\sigma}(\text { iso })-L_{F}^{\sigma} \text { (aniso }\right) .
\end{array}\right.
$$

En l'absence d'irradiation laser ou en champ magnétique fort, nous avons :

$$
L_{F / l}^{\sigma}=L_{F \perp}^{\sigma}=L_{F}^{\sigma} \text { (iso). }
$$

Supposons d'abord le laser assez faible pour que nous puissions négliger les termes non linéaires (ordre de perturbation supérieur à 2). Dans ces conditions, les formules (I.47) montrent que ${ }_{h}^{(2)} \rho_{0}^{0}$ et ${ }_{b}^{(2)} \rho_{0}^{2}$ ne dépendent pas du champ magnétique tant que $\omega_{b} \ll \Delta \nu$ (largeur Doppler). Donc, seul le terme anisotrope dépend du champ magnétique :

$$
{ }^{(2)} L_{F, b g}^{\sigma}(\text { aniso }) \propto \frac{\Gamma_{b}(2)}{\Gamma_{b}^{2}(2)+4 \omega_{b}^{2}} .
$$

Ge terme varie en fonction de $\omega_{\mathrm{b}}$ suivant une courbe de Lorentz dont la largeur à mi-hauteur,
$\Delta \omega_{b}=g_{\mathrm{b}} \beta \Delta H$ est égale à $\Gamma_{b}(2)$. Il suffit donc de tracer expérimentalement la courbe d'effet Hanle, $L_{F / l}^{\sigma}$ (ou $L_{F \perp}^{\sigma}$ ) en fonction de $H$ pour mesurer $\Gamma_{b}(2)$.

Sur la même courbe, il est également facile de mesurer la hauteur relative de l'effet Hanle que nous définissons comme le rapport de la hauteur de la courbe de Lorentz à la variation $\Delta L_{\%}^{\sigma}$ provoquée par le laser en champ magnétique fort :

$R_{/ l \text { ou } \perp}=\left(\frac{L_{F}^{\sigma}\left(\omega_{b}=0\right)-L_{F}^{\sigma}\left(\omega_{b}=\infty\right)}{\Delta L_{F}^{\sigma}\left(\omega_{b}=\infty\right)}\right)_{\| \text {ou } \perp}$

(par $\omega_{b}=\infty$, on entend : $\Gamma_{b}(2) \ll \omega_{b} \ll \Delta v$ ).

Puisque nous nous limitons ici au deuxième ordre, nous avons : $R_{l l}=-R_{\perp}$ et la valeur absolue de ces quantités est égale au rapport $R^{(1)}$ entre la partie anisotrope (pour $H=0$ ) et la partie isotrope de ${ }^{(2)} L_{F}^{\sigma}$ :

$$
R^{(1)}=\frac{{ }^{(2)} L_{F}^{\sigma}(\text { aniso })\left(\omega_{b}=0\right)}{{ }^{(2)} L_{F}^{\sigma}(\text { iso })}
$$

$R^{(1)}$ représente physiquement le rapport entre l'effet d'interférence des composantes $\sigma^{+}$et $\sigma^{-}$de l'onde laser (excitation « $\sigma$ cohérente » qui crée de l'alignement transversal) et l'effet de ces deux composantes séparément (excitation "incohérente " qui modifie les populations et crée de l'alignement longitudinal). En utilisant $(\mathrm{I} .52)$ et $(\mathrm{I} .47 b)$ :

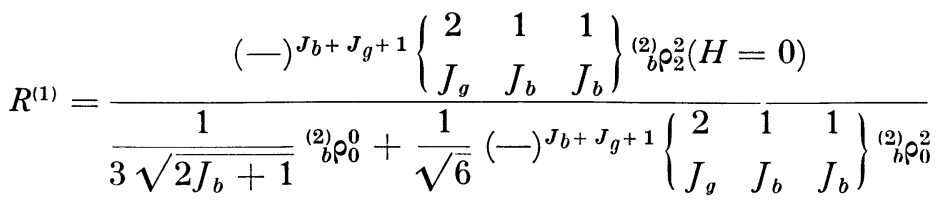



La détermination de $R^{(1)}$ nous donne donc une nouvelle mesure du rapport $\Gamma_{b}(2) / \Gamma_{b}(0)$ qui est proportionnel à la quantité $\frac{1}{R^{(1)}}-\frac{1}{3}$.

Les valeurs utiles des coefficients $\{6, J\}$ sont données en appendice.

Malheureusement, le laser est trop intense en général pour que l'on puisse négliger les termes non linéaires (saturations). Les courbes $L_{F^{\prime}(/ / \text { ou } \perp)}^{\sigma}$ en fonction de $H$ ne sont plus aussi faciles à interpréter : l'effet Hanle est déformé par les saturations; aux ordres de perturbation supérieurs à (2), la partie isotrope aussi bien que la partie anisotrope sont fonction du champ magnétique et présentent une allure résonnante en champ nul [5].

Il en résulte que $\left|R_{\mu l}\right|$ et $\left|R_{\perp}\right|$ ne sont plus égaux et différents de $R^{(1)}$. Pour obtenir $R^{(1)}$, il faudra mesurer $R_{/ /}$et $R_{\perp}$ en fonction de l'intensité du laser $i_{\lambda}$ et chercher la valeur limite commune à ces deux quantités quand $i_{\lambda}$ tend vers zéro.

Nous verrons qu'il est expérimentalement plus commode de construire les deux quantités :

$$
\begin{aligned}
& R=\frac{1}{2}\left[R_{\|}-R_{\perp}\right] \\
& =\frac{1}{2} \frac{L_{F \prime \prime}\left(\omega_{b}=0\right)-L_{F \perp}\left(\omega_{b}=0\right)}{\Delta L_{F}^{\sigma}\left(\omega_{b}=\infty\right)}=\frac{L_{F}^{\sigma}(\text { aniso })\left(\omega_{b}=0\right)}{\Delta L^{\sigma}(\text { iso })\left(\omega_{b}=\infty\right)} \\
& R^{\prime}=\left(\frac{L_{F / I}^{\sigma}-L_{F \perp}^{\sigma}}{\Delta L_{F / I}^{\sigma}+\Delta L_{F \perp}^{\sigma}}\right)_{\omega_{l}=0} \\
& =\frac{L_{F}^{\sigma}(\text { aniso })\left(\omega_{b}=0\right)}{\Delta L_{F}^{\sigma}(\text { iso })\left(\omega_{b}=0\right)}=\frac{R_{/ /}-R_{\perp}}{R_{/ /}+R_{\perp}+2}
\end{aligned}
$$






FIG. 3. - Les différentes grandeurs $R$ :

$$
\begin{gathered}
R_{\|}=\frac{\mathrm{S}_{\|} \mathrm{A}-\mathrm{DC}}{\mathrm{DC}} ; \quad R_{\perp}=\frac{\mathrm{S}_{\perp} \mathrm{A}-\mathrm{DC}}{\mathrm{DC}} \\
R=\frac{\mathrm{S}_{\|} \mathrm{A}-\mathrm{S}_{\perp} \mathrm{A}}{2 \mathrm{DC}}=\frac{\mathrm{S}_{\|} \mathrm{B}}{\mathrm{DC}} \\
R^{\prime}=\frac{\mathrm{S}_{\|} \mathrm{A}-\mathrm{S}_{\perp} \mathrm{A}}{\mathrm{S}_{\|} \mathrm{A}+\mathrm{S}_{\perp} \mathrm{A}}=\frac{\mathrm{S}_{\|} \mathrm{B}}{\mathrm{BA}} .
\end{gathered}
$$

$R^{\prime}$ est le taux d'anisotropie en champ nul, $R$ et $R^{\prime}$ tendent tous deux vers $R^{(1)}$ aux faibles intensités du laser. La figure 3 visualise la définition des grandeurs $R$.
De même, pour mesurer $\Gamma_{b}(2)$ à partir de la courbe d'effet Hanle, il faudra mesurer la largeur $\Delta H$ de cette courbe en fonction de l'intensité du laser et chercher sa limite $\Delta H^{(1)}$ quand $i_{\lambda}$ tend vers 0 :

$$
\Gamma_{b}(2)=g_{b} \beta \Delta H^{(1)}=\operatorname{limite}_{i_{\lambda} \rightarrow 0} g_{b} \beta \Delta H .
$$

Là encore, nous verrons qu'il est utile d'éliminer la partie isotrope de la lumière de fluorescence avant de faire ces mesures. Nous tracerons donc les courbes $L_{F / I}^{\sigma}-L_{F \perp}^{\sigma}$ en fonction de $H$ (qui représente l'effet Hanle proprement dit).

3. GAS DU NIVEAU INFÉRIEUR DE LA TRANSITION LASER. - Nous avons défini la méthode de mesure de $F^{(1)}\left(b g g^{\prime}\right), \Delta H_{b}^{(1)}$ et $R_{b g}^{(1)}$ pour des raies de fluorescences issues du niveau supérieur $b$. La même méthode de mesure peut être appliquée aux raies issues du niveau $a$. Nous obtenons alors des grandeurs $F^{(1)}\left(a f f^{\prime}\right)$, $\Delta H_{a}^{(1)}$ et $R_{a f}^{(1)}$, mais ces quantités ne sont pas aussi simples à interpréter à cause des effets de l'émission spontanée de $b$ vers $a$.

Pour obtenir l'expression de $F^{(1)}\left(a f f^{\prime}\right)$ et $R_{a f}^{(1)}$ à partir expressions (I.58) et (I.66) de $F^{(1)}\left(b g g^{\prime}\right)$ et $R_{b g}^{(1)}$, il faut non seulement échanger les lettres, mais aussi, comme le montre la comparaison des expressions (I.47 a) et $(\mathrm{I} .47 b)$, faire les remplacements :

$$
\begin{aligned}
& \Gamma_{b}(0) \Rightarrow \Gamma_{a}(0)\left[1-\frac{\gamma_{b a}}{\Gamma_{b}(0)}\right]^{-1} \\
& \Gamma_{b}(2) \Rightarrow \Gamma_{a}(2)\left[1-\frac{\gamma_{b a}}{\Gamma_{b}(2)}(-)^{J_{a}+J_{b}+1} \frac{\left\{\begin{array}{ccc}
2 & 1 & 1 \\
J_{a} & J_{b} & J_{b}
\end{array}\right\}\left\{\begin{array}{ccc}
2 & J_{b} & J_{b} \\
1 & J_{a} & J_{a}
\end{array}\right\}\left(2 J_{b}+1\right)}{\left\{\begin{array}{lll}
2 & 1 & 1 \\
J_{b} & J_{a} & J_{a}
\end{array}\right\}}\right]^{-1} .
\end{aligned}
$$

Pour obtenir par cette méthode une valeur du rapport $\Gamma_{a}(2) / \Gamma_{a}(0)$, il faudra donc au préalable déterminer $\Gamma_{b}(0), \Gamma_{b}(2)$ et $\gamma_{b a}$.

En ce qui concerne $\Delta H_{a}^{(1)}$, il n'est plus relié de façon simple à $\Gamma_{a}(2)$, car à la courbe de Lorentz ordinaire (de largeur $\Gamma_{a}(2)$ ) s'ajoute une courbe plus compliquée [11], [12] dont la forme dépend à la fois de
$\Gamma_{a}(2)$ et de $\Gamma_{b}(2)$. Heureusement, dans le cas expérimental du niveau $2 p_{4}$ du néon, une simplification importante permettra de négliger cet effet Hanle de transfert.

Appendice. - Les valeurs des coefficients $\{6 J\}$ sont données dans le tableau ci-dessous :

$$
(-)^{J_{a}+J_{b}+1}\left\{\begin{array}{ccc}
2 & 1 & 1 \\
J_{b} & J_{a} & J_{a}
\end{array}\right\}=\mid \begin{array}{cccc}
J_{a} & J_{b}=0 & J_{b}=1 & J_{b}=2 \\
1 & 1 / 3 & -1 / 6 & 1 / 30 \\
2 & 0 & \sqrt{ } \overline{21} / 30 & -\sqrt{21} / 30
\end{array}
$$

ce qui donne pour $(-)^{2 J_{a}+J_{b}+J_{g}}\left\{\begin{array}{ccc}2 & 1 & 1 \\ J_{g} & J_{a} & J_{a}\end{array}\right\}\left\{\begin{array}{ccc}2 & 1 & 1 \\ J_{b} & J_{a} & J_{a}\end{array}\right\}$ :

$$
\begin{array}{rccc}
J_{a}=1: & & & \\
J_{g} & J_{b}=0 & J_{b}=1 & J_{b}=2 \\
0 & 1 / 9 & -1 / 18 & 1 / 90 \\
1 & -1 / 18 & 1 / 36 & -1 / 180 \\
2 & 1 / 90 & -1 / 180 & 1 / 900
\end{array}
$$

$$
\begin{aligned}
& J_{a}=2 \text { : } \\
& J_{g} \quad J_{b}=1 \quad J_{b}=2 \\
& 1 \quad 21 / 900 \quad-21 / 900 \\
& 2 \quad-21 / 900 \quad 21 / 900
\end{aligned}
$$




\section{BIBLIOGRAPHIE}

[1] Lamb (W. E.), Phys. Rev., 1964, 134, 6 A, 1429.

[2] Javan (A.), Bull. Amer. Phys. Soc., 1964, 9, 489.

[3] Fork (R. L.), Hargrove (L. E.) et Poliack (M. A.), Phys. Rev., Lett., 1964, 12, 705.

[4] Cordover (R. H.), Parks (J.), Szoke (A.) et Javan (A.), "Physics of quantum electronics ", Porto Rico, juin 1965, édité par $P$. Kelley, B. Lax et P. Tannenwald, McGraw-Hill Book Company, 1966, p. 591.

[5] Decomps (B.) et Dumont (M.), C. R. Acad. Sc., 1966, 262 B, 1520.

[6] Decomps (B.) et Dumont (M.), C. R. Acad. Sc., 1967, 265 B, 249.

[7] Bloembergen (N.) et Shein (Y. R.), Phys. Rev., 1964, 133, A 37.
[8] Omoni (A.), J. Physique, 1965, 26, 26.

OMONT (A.), Thèse, Paris, 1967.

Durand (G.), Thèse, Paris.

[9] Omont (A.), J. Physique, 1965, 26, 576.

[10] Saloman (E. B.) et Happer (W.), Phys. Rev., 1966, 144, 7.

[11] Nedelec (O.), Thèse, Grenoble, 1966.

[12] Ducloy (M.), à paraître.

[13] Cohen-TannoudjI (C.), Thèse, Paris, 1961, Annales de Physique, 1962, 7, 423 et 469.

[14] Mitcheli (A. C.) et Zemansky (M. W.), Cambridge University Press, 1934.

[15] Fried (B. D.) et Conte (S. D.), "The plasma dispersion fonction ", Academic Press, New York, Iondon, 1961. 